ISSN 0103-9954

\title{
CARACTERIZAÇÃO ECOLÓGICA DE ESPÉCIES ARBÓREAS OCORRENTES EM AMBIENTES DE MATA CILIAR, COMO SUBSÍDIO À RECOMPOSIÇÃO DE ÁREAS ALTERADAS NAS CABECEIRAS DO RIO GRANDE, MINAS GERAIS, BRASIL ${ }^{1}$
}

\author{
ECOLOGICAL CHARACTERIZATION OF ARBOREAL SPECIES OCURRING IN CILIARY \\ FOREST ENVIRONMENT, AS BASIS FOR THE RECOVERY OF AREAS ALTERED IN THE \\ HEADBOARDS OF RIO GRANDE, MINAS GERAIS, BRAZIL ${ }^{1}$
}

Israel Marinho Pereira ${ }^{2}$ Soraya Alvarenga Botelho ${ }^{3}$ Eduardo van den $\mathrm{Berg}^{4}$

Ary Teixeira de Oliveira-Filho ${ }^{5}$ Evandro Luiz Mendonça Machado ${ }^{6}$

\begin{abstract}
RESUMO
Este estudo teve como objetivo verificar as diferenças na composição da flora e das guildas das comunidades arbustivo-arbóreas em três áreas de mata ciliar em diferentes estágios de sucessão florestal. Foram alocadas 46 parcelas de $400 \mathrm{~m}^{2}$ e todos os indivíduos arbóreos existentes no seu interior, com diâmetro à altura do peito (DAP) $=5 \mathrm{~cm}$, foram amostrados. Foram identificadas 282 espécies distribuídas em 140 gêneros e 61 famílias. No que se refere às guildas de dispersão, constatou-se que, em média, $72 \%$ das espécies e $59 \%$ dos indivíduos têm suas fontes de propágulos disseminadas por animais (zoocórica), 19\% e 23\% pelo vento (anemocórica) e 6\% e $13 \%$ por meio barocoria ou balística (autocórica). As proporções de espécies e indivíduos por grupo ecológico foram, respectivamente, pioneiras: $14,5 \%$ e $18 \%$; clímax exigente de luz: 49,5\% e $62,5 \%$ e, clímax tolerante à sombra: 36,5\% e 21,5\%.Verificou-se, ainda, que houve um aumento significativo da importância das espécies zoocóricas e tolerantes à sombra com a maturidade das florestas, enquanto o número de espécies que necessitam de luz com dispersão abiótica decresceu significativamente com o avanço dessa maturidade.
\end{abstract}

Palavras-chave: mata ciliar; dispersão de propágulos; grupo ecológico; floresta secundária.

\begin{abstract}
The objective of this study was to verify the differences in the composition of the flora and of guilds of arbustivearboreal communities of three areas of ciliary forest, in different forest succession stages. Fourty-six plots of 400 $\mathrm{m}^{2}$ were allocated and all existent arboreal individuals inside the portions with diameter breast height (DBH) greater than or equal to $5 \mathrm{~cm}$ were sampled. A total of 282 species, distributed in 140 genera and 61 families were identified. With regard to dispersal guilds, it was found that on average, the spread of sources of propagules of $72 \%$ of the species and $59 \%$ of the individuals was zoochorous, while $19 \%$ and $23 \%$, respectively, was wind anemocoric, and $6 \%$ and $13 \%$, respectively, was autochoric. The proportions of individuals per species and ecological group were, respectively, pioneers: $14.5 \%$ and $18 \%$; climax demanding of light: $49.5 \%$ and $62.5 \%$, and shade-tolerant: $36.5 \%$ and $21.5 \%$. It was also verified that there was a significant increase in the importance of animal dispersed species and shade tolerant species with the maturity of forests, while the number of species that required light abiotically dispersed decreased significantly with the advancement of maturity.
\end{abstract}

Keywords: ciliary forest; dispersion of propagules; ecological group; secondary forest.

1. Parte da tese de doutorado do primeiro autor.

2. Engenheiro Florestal, Dr., Professor do Departamento de Engenharia Florestal, Universidade Federal dos Vales do Jequitinhonha e Mucuri, CEP 39100-000, Diamantina (MG). imarinhopereira@gmail.com

3. Engenheira Florestal, Dr ${ }^{a}$., Professora do Departamento de Ciências Florestais, Universidade Federal de Lavras, Caixa Postal, 3037, CEP 37200, Lavras (MG). sbotelho@ufla.br

4. Engenheiro Agrônomo, Dr., Professor do Departamento de Biologia, Universidade Federal de Lavras, Caixa Postal 37, CEP 37200-000, Lavras (MG). evandenb@ufla.br

5. Engenheiro Florestal, Dr. Professor do Departamento de Botânica do Instituto de Ciências Biológicas, Universidade Federal de Minas Gerais, Av. Antônio Carlos, 66627, Pampulha CEP 31270-901, Belo Horizonte (MG). ary@icb.ufmg.br

6. Engenheiro Florestal, Dr., Professor do Departamento de Engenharia Florestal, Universidade Federal dos Vales do Jequitinhonha e Mucuri, CEP 39100-000, Diamantina (MG). machadoelm@gmail.com

Recebido para publicação em 12/06/2008 e aceito em 29/04/2010.

Ci. Fl., v. 20, n. 2, abr.-jun., 2010 


\section{INTRODUÇÃO}

A utilização inadequada dos recursos naturais tem causado efeitos negativos aos ecossistemas no mundo inteiro. Entre os ecossistemas mais perturbados e/ou degradados pelo homem, destacam-se as matas ciliares. No entanto, elas estão relacionadas no art. $2^{\circ}$, da Lei n. 4.771/65, que estabelece o Código Florestal Brasileiro no qual são contempladas como áreas de preservação permanente "as florestas e demais formas de vegetação existentes no entorno dos rios, lagos, nascentes, lagoas e reservatórios", especificando, na maioria das situações, a dimensão mínima da faixa marginal que deve ser preservada.

A importância da preservação ou restauração das florestas ao longo dos rios e ao redor de lagos, reservatórios e nascentes fundamenta-se no amplo aspecto de benefícios que essa vegetação traz ao ecossistema, exercendo função protetora sobre os recursos naturais bióticos e abióticos (DURIGAN e SILVEIRA, 1999). Para Marques et al. (1961), a proteção eficaz que a mata ciliar exerce contra o assoreamento que ocorre em função de diversos tipos de erosão, por si só constitui uma valiosa contribuição à preservação desse recurso natural; serve de hábitat para a fauna silvestre, ao proporcionar água, alimento e abrigo para várias espécies terrestres e aquáticas; funciona como corredor de fauna entre fragmentos florestais; além de proporcionar o fluxo gênico entre as populações (BOTELHO e DAVIDE, 2002).

A implantação ou a recomposição de matas ciliares requer o emprego de técnicas adequadas, geralmente definidas em virtude de avaliações detalhadas das condições locais e da utilização dos conhecimentos científicos existentes. A obtenção de informações ecológicas e silviculturais tais como, exigência de luz, relação planta-animais, frequência e densidade natural pode levar ao conhecimento da combinação ideal de espécies dentro dos mosaicos de estádios sucessionais, semelhantes aos formados nas florestas tropicais naturais. A tentativa de separação de espécies em diferentes grupos quanto à sucessão secundária tem sido defendida por diversos autores (DENSLOW, 1980a; WHITMORE, 1982).

A interação entre a fauna e a vegetação é fundamental no processo de recuperação de áreas degradadas e deve ser considerada durante o planejamento de reabilitação dessas áreas (GUEDES et al., 1997). Segundo Angel-de-Oliveira (1998), a maioria das espécies arbustivo-arbóreas características das florestas tropicais tem seus diásporos disseminados por animais. As sementes têm papel fundamental na regeneração natural das florestas e a dispersão é um fator importante na determinação de padrões de deposição dessas sementes (MARTINEZ - RAMOS e SOTO CASTRO, 1993; LOISELLE et al., 1996).

Diante do exposto, na presente pesquisa objetivou-se: i) conhecer o compartimento arbustivo-arbóreo de dois ambientes de mata ciliar (aluvião e encosta) em diferentes estádios de regeneração na região das cabeceiras do rio Grande; ii) conhecer os padrões ecológicos relacionados às guildas de regeneração, dispersão e estratificação e correlacioná-los com o estágio sucessional das áreas e os diferentes ambientes estudados, visando à seleção de espécies potenciais para serem utilizadas na recomposição da cobertura vegetal das porções degradadas das cabeceiras do rio Grande.

\section{MATERIAL E MÉTODOS}

\section{Caracterização e localização das áreas de estudo}

Para a realização deste trabalho, foram estudadas três áreas de mata ciliar, assim caracterizadas: aluvião $=$ fragmento de mata ciliar sobre solo de aluvião com aproximadamente 3 ha, em regeneração há 50 anos, situado às margens direita e esquerda do rio Grande, a cerca de $15 \mathrm{~km}$ a jusante da nascente principal $\left(22^{\circ} 10^{\prime} \mathrm{S}\right.$ e $\left.44^{\circ} 28^{\prime} \mathrm{W}\right)$ a 1.150 $\mathrm{m}$ de altitude; encosta em regeneração $=$ fragmento de mata ciliar de encosta com aproximadamente 15 ha, em estágio intermediário de sucessão ecológica, localizado às margens do rio Grande, a cerca de 5 $\mathrm{km}$ a jusante de sua nascente principal $\left(22^{\circ} 14\right.$ 'S e $44^{\circ} 34^{\prime} \mathrm{W}$ ) a uma altitude de 1.500 a $1.580 \mathrm{~m}$; e encosta conservada $=$ fragmento de mata ciliar de encosta conservada de aproximadamente 10 ha, localizado às margens do rio Grande, a cerca de 10 $\mathrm{km}$ a jusante de sua nascente principal $\left(22^{\circ} 13^{\prime} \mathrm{S}\right.$ e $44^{\circ} 32^{\prime} \mathrm{W}$ ) e a uma altitude em torno de $1.300 \mathrm{~m}$.

O clima da região é do tipo Cfa de Köppen (úmido subtropical) e a temperatura média anual é de $16,7^{\circ} \mathrm{C}$, com médias mensais variando de $13,0^{\circ} \mathrm{C}$ (julho) a $19,7^{\circ} \mathrm{C}$ (janeiro). A precipitação média anual é de $2.108 \mathrm{~mm}$, com médias mensais variando de $341 \mathrm{~mm}$, no trimestre mais chuvoso (dezembro a fevereiro), a $35 \mathrm{~mm}$, no trimestre mais seco (junho a agosto). 


\section{Composição da comunidade arbustivo-arbórea}

O levantamento da comunidade arbórea foi realizado no período de maio a julho de 2004 em 46 parcelas de $400 \mathrm{~m}^{2}$, utilizadas para o estudo da estrutura fitossociológica, além de caminhamentos de varredura nas áreas em busca de espécies não registradas nas parcelas. Nas áreas de aluvião e encosta em regeneração foram plotadas dez parcelas de 10×40 m. Já na área de encosta conservada foram plotadas 26 parcelas de $20 \times 20 \mathrm{~m}$. Para as duas áreas de encosta, as parcelas obedeceram ao gradiente de altitude, sendo plotadas parcelas na encosta inferior próxima ao leito do rio, na meia encosta e alta encosta (topo). Alguns indivíduos foram identificados no campo, enquanto outros tiveram material botânico coletado para posterior identificação. Todos os indivíduos arbóreos vivos com circunferência à altura do peito (CAP) maior ou igual a $15,7 \mathrm{~cm}$, encontrados no interior das parcelas, foram registrados e identificados.

\section{Caracterização ecológica das espécies:}

Para comparar os diferentes habitats quanto ao perfil de estratégias ecológicas de suas espécies arbóreas, estas foram classificadas em guildas, de acordo com as estratégias de regeneração, estratificação e dispersão.

Guildas de regeneração são grupos de espécies que se regeneram em ambiente com mesma luminosidade (DESLOW, 1980b). Na classificação das espécies em guildas de regeneração, adotou-se a definição proposta por Swaine \& Whitmore (1988), reunindo-as em três grupos: pioneiras, aquelas que necessitam de luz direta para germinar e se estabelecer; clímax exigente de luz, aquelas cujas sementes conseguem germinar nas condições de sombra do sub-bosque, embora os imaturos necessitem de luz abundante para crescer e atingir o dossel, e clímax tolerante à sombra, aquelas que germinam e conseguem crescer nas condições de sombra do sub-bosque, atingindo a maturidade sob o próprio dossel ou após atingir o dossel da floresta, conforme a espécie.

Guildas de dispersão são grupos de espécies que utilizam os mesmos agentes dispersores (WHEELWRIGHT, 1985). Quanto à guilda de dispersão, as espécies foram classificadas de acordo com Van der Pijl (1982), nas categorias: anemocóricas, aquelas cujas sementes são disseminadas pelo vento; zoocóricas, espécies que apresentam características que indicam que a dispersão de suas sementes ou propágulos é realizada por animais, e autocóricas, aquelas espécies que dispersam suas sementes pela gravidade ou por deiscência explosiva. Para a obtenção de informações ecológicas sobre as espécies, foram utilizadas informações encontradas na literatura (MORELLETO et al., 1989; MORELLATO e LEITÃO FILHO, 1992; PENHALBER e MANTOVANI, 1997; CARMO e MORELLATO, 2001; GARCIA e PIRANI, 2001; LORENZI, 2002a; LORENZI, 2002b; NUNES et al., 2003), acrescidas do conhecimento dos próprios autores. As espécies pertencentes ao grupo das Pteridófitas foram classificadas como "outras".

Guildas de estratificação são grupos de espécies que, quando adultas, ocupam o mesmo estrato da vegetação (WILSON, 1989). A classificação das espécies com relação às guildas de estratificação foi baseada na altura que cada espécie normalmente alcança na maturidade e utilizando as mesmas categorias e intervalos definidos por Oliveira-Filho et al. (1997) para florestas da região: pequenas, espécies com altura inferior a $8 \mathrm{~m}(2,0$ a 7,9 m); médias, espécies que, na maturidade, alcançam alturas de 8,0 a 17,5 m; e grandes, aquelas espécies que atingem alturas superiores a 17,5 m.

Para verificar a distribuição das frequências de espécies arbóreas nas guildas de regeneração, estratificação e dispersão foram feitas tabelas de contingência, cruzando-se a frequência de espécies nas guildas de regeneração versus dispersão, regeneração versus estratificação e estratificação versus dispersão. Aplicou-se também o teste de quiquadrado para tabelas de contingência (ZAR, 1996).

\section{RESULTADOS E DISCUSSÃO}

Foram identificadas, no total, 282 espécies, distribuídas em 140 gêneros e 61 famílias (Tabela1). $\mathrm{Na}$ área de aluvião, foram amostrados 1.206 indivíduos, distribuídos em 81 espécies, 57 gêneros e 29 famílias. Na área de encosta em regeneração foram registrados 897 indivíduos, pertencentes a 118 espécies, 71 gêneros e 41 famílias. Já na encosta conservada foram encontrados 2.553 indivíduos, 215 espécies, 120 gêneros e 56 famílias. A relação das espécies registradas nas três áreas e suas guildas encontram-se na Tabela 1. 
Pereira, I. M. et al.

TABELA 1: Relação das espécies arbóreas registradas em três remanescentes de mata ciliar, em Bocaina de Minas, MG, dispostas em ordem alfabética de família, seguidas de suas guildas.

TABLE 1: Relationship of the arboreal species registered in three remainders of ciliary forest, in Bocaina of Mines, MG, arranged in alphabetical order of family, followed by their guilds.

\begin{tabular}{|c|c|c|c|c|c|c|c|c|c|c|}
\hline \multirow{2}{*}{ Família/Espécie } & \multirow{2}{*}{$\mathrm{Hab}$} & \multicolumn{3}{|c|}{ Áreas } & \multicolumn{3}{|c|}{ Guildas } & \multicolumn{2}{|c|}{ Fruto } & \multirow{2}{*}{$\mathrm{AD}$} \\
\hline & & I & II & III & Reg & Estr & Disp & Tipo & Cor & \\
\hline ANACARDIACEAE & & & & & & & & & & \\
\hline Tapirira guianensis Aubl. & AR & 0 & 0 & 6 & $\mathrm{CL}$ & Méd & Zoo & Drupa & Preto & $\mathrm{A}$ e $\mathrm{M}$ \\
\hline $\begin{array}{l}\text { Tapirira obtusa (Benth.) J.D.Mitch } \\
\text { ANNONACEAE }\end{array}$ & AR & 0 & 0 & 25 & $\mathrm{CL}$ & Grd & Zoo & Drupa & Preto & $\mathrm{A}$ e $\mathrm{M}$ \\
\hline Annona cacans Warm. & $\mathrm{AR}$ & 5 & 0 & 10 & $\mathrm{CL}$ & Méd & Zoo & Sincarpo & Amarelado & M \\
\hline $\begin{array}{l}\text { Annona dolabripetala (Raddi) } \\
\text { H.Rainer }\end{array}$ & AR & 0 & 0 & Flr & $\mathrm{CL}$ & Grd & Zoo & Sincarpo & Preto & M \\
\hline $\begin{array}{l}\text { Annona emarginata (Schltdl.) } \\
\text { H.Rainer }\end{array}$ & AR & 40 & 0 & 0 & $\mathrm{CL}$ & Méd & Zoo & Sincarpo & Amarelo & M \\
\hline Annona sylvatica A.St.-Hil. & AR & 0 & 0 & 8 & $\mathrm{CL}$ & Méd & Zoo & Sincarpo & Amarelo & M \\
\hline $\begin{array}{l}\text { Cymbopetalum brasiliense (Vell.) } \\
\text { Benth. ex Baill. }\end{array}$ & AV & 0 & 0 & 4 & $\mathrm{CS}$ & Méd & Zoo & Drupa & Vermelho & A \\
\hline Duguetia salicifolia R.E.Fr. & AR & 0 & 1 & 4 & CS & Grd & Zoo & Sincarpo & Vermelho & M \\
\hline Guatteria australis A.St.-Hil. & $\mathrm{AR}$ & 0 & 20 & 21 & CS & Méd & Zoo & Drupa & Preto & A e $M$ \\
\hline Guatteria latifolia (Mart.) R.E.Fr. & $\mathrm{AR}$ & 0 & 5 & 4 & $\mathrm{CS}$ & Méd & Zoo & Drupa & Preto & A \\
\hline Guatteria pohliana Schltdl. & AR & 0 & 0 & 79 & $\mathrm{CS}$ & Méd & Zoo & Drupa & Roxa & $\mathrm{A}$ e $\mathrm{M}$ \\
\hline $\begin{array}{l}\text { Xylopia brasiliensis Spreng. } \\
\text { APOCYNACEAE }\end{array}$ & $\mathrm{AR}$ & 0 & 0 & Flr & $\mathrm{CS}$ & Grd & Zoo & Cápsula & Bicolor & A \\
\hline Aspidosperma australe Müll.Arg. & AR & 0 & 0 & Flr & $\mathrm{CL}$ & Méd & Ane & Folículo & Bicolor & $\mathrm{V}$ \\
\hline Aspidosperma parvifolium A.DC. & AR & 1 & 1 & 2 & $\mathrm{CL}$ & Méd & Ane & Folículo & Paleáceo & $\mathrm{V}$ \\
\hline $\begin{array}{l}\text { Aspidosperma spruceanum Benth. } \\
\text { ex Müll.Arg. } \\
\text { AQUIFOLIACEAE }\end{array}$ & $\mathrm{AR}$ & 0 & 0 & 3 & $\mathrm{CL}$ & Grd & Ane & Folículo & Paleáceo & $\mathrm{V}$ \\
\hline Ilex affinis Gardner & AR & 0 & 1 & 2 & $\mathrm{CL}$ & Méd & Zoo & Drupa & Preto & A \\
\hline Ilex cerasifolia Reissek & AR & 0 & 0 & 1 & $\mathrm{CS}$ & Méd & Zoo & Baga & Vermelho & A \\
\hline Ilex dumosa Reissek & $\mathrm{AB}$ & 3 & 0 & 0 & CL & Méd & Zoo & Drupa & Preto & A \\
\hline Ilex paraguariensis A.St.-Hil. & $\mathrm{AR}$ & 0 & 1 & 0 & $\mathrm{CL}$ & Méd & Zoo & Drupa & Vermelho & A \\
\hline $\begin{array}{l}\text { Ilex theezans Mart. ex Reissek } \\
\text { ARALIACEAE }\end{array}$ & $\mathrm{AR}$ & 21 & 0 & Flr & $\mathrm{CL}$ & Grd & Zoo & Drupa & Preto & A \\
\hline $\begin{array}{l}\text { Oreopanax capitatus (Jacquin) } \\
\text { Decne \& Planchon }\end{array}$ & $\mathrm{AR}$ & 0 & 0 & 1 & $\mathrm{CS}$ & Méd & Zoo & Baga & Arroxeado & $\mathrm{A}$ \\
\hline $\begin{array}{l}\text { Schefflera calva (Cham.) Frodin \& } \\
\text { Fiaschi }\end{array}$ & AR & 0 & 2 & 22 & $\mathrm{CS}$ & Méd & Zoo & Baga & Verde & \\
\hline $\begin{array}{l}\text { ARAUCARIACEAE } \\
\text { Araucaria angustifolia (Bert.) } \\
\text { Kuntze } \\
\text { ARECACEAE }\end{array}$ & $\mathrm{AR}$ & 0 & Flr & Flr & $\mathrm{CL}$ & Grd & Zoo & Pinhão & Marrom & AV \\
\hline Geonoma schottiana Mart. & $\mathrm{AB}$ & 0 & 0 & 2 & $\mathrm{CS}$ & Peq & Zoo & Drupa & Preto & A \\
\hline $\begin{array}{l}\text { Syagrus romanzoffiana (Cham.) } \\
\text { Glassman } \\
\text { ASTERACEAE }\end{array}$ & $\mathrm{AR}$ & 3 & 0 & 0 & $\mathrm{CL}$ & Grd & Zoo & Drupa & Laranja & A e $M$ \\
\hline Austrocritonia velutina (Gardn.) & $\mathrm{AR}$ & 1 & 0 & 0 & CL & Méd & Ane & Cinsela & Amarelo & $\mathrm{V}$ \\
\hline
\end{tabular}

Continua $\ldots$

Ci. Fl., v. 20, n. 2, abr.-jun., 2010 
TABELA 1: Continuação ...

TABLE 1: Continued ...

\begin{tabular}{|c|c|c|c|c|c|c|c|c|c|c|}
\hline \multirow{2}{*}{ Família/Espécie } & \multirow{2}{*}{$\mathrm{Hab}$} & \multicolumn{3}{|c|}{ Áreas } & \multicolumn{3}{|c|}{ Guildas } & \multicolumn{2}{|c|}{ Fruto } & \multirow{2}{*}{$\mathrm{AD}$} \\
\hline & & I & II & III & Reg & Estr & Disp & Tipo & Cor & \\
\hline R.M.King \& H.Rob. & & & & & & & & & & \\
\hline $\begin{array}{l}\text { Baccharis oblongifolia (Ruiz \& } \\
\text { Pav.) Pers. }\end{array}$ & $\mathrm{AB}$ & 1 & 1 & 0 & $\mathrm{P}$ & Méd & Ane & Cipsela & Amarelo & $\mathrm{V}$ \\
\hline Baccharis serrulata Pers. & $\mathrm{AB}$ & 0 & 2 & 1 & $\mathrm{P}$ & Méd & Ane & Cipsela & Amarelo & $\mathrm{V}$ \\
\hline $\begin{array}{l}\text { Dasyphyllum brasiliense (Spreng.) } \\
\text { Cabrera }\end{array}$ & $\mathrm{AB}$ & 0 & 1 & 0 & CL & Grd & Zoo & Cipsela & Amarelo & $\mathrm{V}$ \\
\hline $\begin{array}{l}\text { Eremanthus erythropappus (DC.) } \\
\text { MacLeish }\end{array}$ & $\mathrm{AB}$ & 0 & 24 & Flr & $\mathrm{P}$ & Méd & Ane & Cipsela & Amarelo & $\mathrm{V}$ \\
\hline $\begin{array}{l}\text { Gochnatia paniculata (Less.) } \\
\text { Cabrera }\end{array}$ & $\mathrm{AB}$ & 0 & Flr & Flr & $\mathrm{P}$ & Peq & Ane & Cipsela & Preto & V \\
\hline $\begin{array}{l}\text { Heterocondylus vauthierianus } \\
\text { (DC.) R.M.King \& H.Rob. }\end{array}$ & AR & 0 & 8 & 0 & $\mathrm{P}$ & Méd & Ane & Cipsela & & $\mathrm{V}$ \\
\hline Piptocarpha macropoda Baker & AR & 0 & 5 & 10 & $\mathrm{P}$ & Méd & Ane & Cipsela & Marron & V \\
\hline $\begin{array}{l}\text { Piptocarpha regnellii (Sch.Bip.) } \\
\text { Cabrera }\end{array}$ & $\mathrm{AR}$ & 0 & 41 & 1 & $\mathrm{P}$ & Méd & Ane & Cipsela & Marron & $\mathrm{V}$ \\
\hline Stifftia chrysantha Mikan & $\mathrm{AR}$ & 0 & 0 & Flr & $\mathrm{CS}$ & Peq & Ane & Cipsela & Marron & $\mathrm{V}$ \\
\hline $\begin{array}{l}\text { Vernonanthura divaricata (Spreng.) } \\
\text { H.Rob. }\end{array}$ & AR & 1 & 8 & 41 & $\mathrm{P}$ & Grd & Ane & Cipsela & Branco & $\mathrm{V}$ \\
\hline $\begin{array}{l}\text { Vernonanthura discolor (Spreng.) } \\
\text { H.Rob. } \\
\text { BIGNONIACEAE }\end{array}$ & AR & 0 & 13 & 28 & $\mathrm{P}$ & Grd & Ane & Cipsela & Branco & V \\
\hline $\begin{array}{l}\text { Cybistax antisyphilitica (Mart.) } \\
\text { Mart. }\end{array}$ & AR & Flr & 0 & Flr & $\mathrm{CL}$ & Peq & Ane & Cápsula & $\begin{array}{l}\text { Castanho- } \\
\text { escura }\end{array}$ & $\mathrm{V}$ \\
\hline $\begin{array}{l}\text { Handroanthus albus (Cham.) } \\
\text { Mattos }\end{array}$ & AR & 0 & 2 & 0 & $\mathrm{CL}$ & Grd & Ane & Síliqua & Amarelo & V \\
\hline $\begin{array}{l}\text { Handroanthus bureavii (Sandwith) } \\
\text { S.O.Grose }\end{array}$ & AR & 6 & 0 & 0 & $\mathrm{CL}$ & Grd & Ane & Síliqua & Preto & V \\
\hline $\begin{array}{l}\text { Handroanthus chrysotrichus (Mart. } \\
\text { ex A.DC.) Mattos }\end{array}$ & AR & 0 & 0 & 3 & $\mathrm{CL}$ & Méd & Ane & Síliqua & $\begin{array}{l}\text { Verde - } \\
\text { musgo }\end{array}$ & $\mathrm{V}$ \\
\hline $\begin{array}{l}\text { Handroanthus vellosoi (Toledo) } \\
\text { Mattos }\end{array}$ & AR & 0 & 0 & 5 & $\mathrm{CL}$ & Grd & Ane & Cápsula & Marron & $\mathrm{V}$ \\
\hline $\begin{array}{l}\text { Jacaranda macrantha Cham. } \\
\text { CANELLACEAE }\end{array}$ & AR & 0 & 0 & 1 & $\mathrm{CL}$ & Grd & Ane & Cápsula & Cinza-escuro & V \\
\hline $\begin{array}{l}\text { Capsicodendron dinisii (Schwacke) } \\
\text { Occhioni } \\
\text { CELASTRACEAE }\end{array}$ & AR & 2 & 0 & 0 & $\mathrm{CS}$ & Grd & Zoo & Baga & Vermelho & $\mathrm{A}$ e $\mathrm{M}$ \\
\hline Maytenus communis Reissek & AR & 0 & 0 & 1 & $\mathrm{CS}$ & Méd & Zoo & Cápsula & Bicolor & A \\
\hline Maytenus evonymoides Reissek & AR & 0 & Flr & 0 & $\mathrm{CS}$ & Peq & Zoo & Cápsula & Bicolor & $\mathrm{A}$ \\
\hline Maytenus gonoclada Mart. & AR & 1 & 2 & 0 & $\mathrm{CS}$ & Méd & Zoo & Cápsula & Marron & A \\
\hline $\begin{array}{l}\text { Maytenus salicifolia } \text { Reissek } \\
\text { CHLORANTHACEAE }\end{array}$ & AR & 0 & 0 & 3 & $\mathrm{CS}$ & Méd & Zoo & Cápsula & Bicolor & $\mathrm{A}$ \\
\hline $\begin{array}{l}\text { Hedyosmum brasiliense Mart. } \\
\text { CLETHRACEAE }\end{array}$ & AR & Flr & Flr & Flr & $\mathrm{CL}$ & Peq & zoo & & Branco & A \\
\hline $\begin{array}{l}\text { Clethra scabra Pers. } \\
\text { CLUSIACEAE }\end{array}$ & AR & 0 & 40 & 34 & CL & Méd & Ane & Cápsula & Marron & $\mathrm{V}$ \\
\hline Clusia criuva Cambess. & $\mathrm{AR}$ & 0 & 0 & Flr & $\mathrm{CS}$ & Méd & Zoo & Cápsula & Vermelho & A \\
\hline Clusia fragrans Gardn. & AR & 0 & 0 & 2 & $\mathrm{CS}$ & Méd & Zoo & Cápsula & Bicolor & A \\
\hline $\begin{array}{l}\text { Tovomita paniculata (Spreng.) } \\
\text { Cambess. } \\
\text { CORDIACEAE }\end{array}$ & $\mathrm{AB}$ & 0 & 0 & 12 & $\mathrm{CS}$ & Méd & Zoo & Cápsula & Vermelho & A \\
\hline Cordia ecalyculata Vell. & AR & 0 & 0 & 7 & $\mathrm{CS}$ & Grd & Zoo & Baga & Vermelho & $\mathrm{A}$ \\
\hline $\begin{array}{l}\text { Cordia superba } \text { Cham. } \\
\text { CUNONIACEAE }\end{array}$ & AR & 0 & 0 & Flr & CL & Méd & Ane & Drupa & Amarelo & A \\
\hline Lamanonia ternata Vell. & AR & 0 & 2 & 84 & $\mathrm{CL}$ & Méd & Ane & Cápsula & Paleáceo & $\mathrm{V}$ \\
\hline $\begin{array}{l}\text { Weinmannia paulliniifolia } \text { Pohl } \\
\text { CYATHEACEAE }\end{array}$ & AR & 0 & 2 & 0 & $\mathrm{CL}$ & Méd & Aut & Cápsula & Paleáceo & BL \\
\hline $\begin{array}{l}\text { Alsophila sternbergii (Sternb.) } \\
\text { Conant }\end{array}$ & AR & 0 & 1 & 20 & CS & Méd & umid & & & \\
\hline Alsophila setosa Kaulf & $\mathrm{AV}$ & 0 & 1 & Flr & $\mathrm{CS}$ & Peq & umid & & & \\
\hline Cyathea atrovirens (Langsd.\& & AV & 0 & 1 & 0 & $\mathrm{CS}$ & Peq & umid & & & \\
\hline
\end{tabular}


Pereira, I. M. et al.

TABELA 1: Continuação ...

TABLE 1: Continued ...

\begin{tabular}{|c|c|c|c|c|c|c|c|c|c|c|}
\hline \multirow{2}{*}{ Família/Espécie } & \multirow{2}{*}{$\mathrm{Hab}$} & \multicolumn{3}{|c|}{ Áreas } & \multicolumn{3}{|c|}{ Guildas } & \multicolumn{2}{|c|}{ Fruto } & \multirow{2}{*}{$\mathrm{AD}$} \\
\hline & & $\mathrm{I}$ & II & III & Reg & Estr & Disp & Tipo & Cor & \\
\hline \multicolumn{11}{|l|}{ Fisch.) Domin } \\
\hline $\begin{array}{l}\text { Cyathea corcovadensis (Raddi) } \\
\text { Domin }\end{array}$ & AV & 0 & 4 & 53 & $\mathrm{CS}$ & Méd & umid & & & \\
\hline Cyathea delgadii Sternb. & AV & 0 & 5 & 59 & $\mathrm{CS}$ & Méd & umid & & & \\
\hline $\begin{array}{l}\text { Cyathea dichromatolepis (Fée) } \\
\text { Domin }\end{array}$ & AV & 0 & 1 & 2 & CS & Méd & umid & & & \\
\hline Cyathea phalerata Mart. & AV & 0 & 6 & 3 & $\mathrm{CS}$ & Méd & umid & & & \\
\hline $\begin{array}{l}\text { Sphaeropteris gardneri (Hook.) } \\
\text { Tryon } \\
\text { DICKSONIACEAE }\end{array}$ & AV & 0 & 3 & Flr & $\mathrm{CS}$ & Peq & umid & & & \\
\hline $\begin{array}{l}\text { Dicksonia sellowiana Hook. } \\
\text { ELAEOCARPACEAE }\end{array}$ & AV & 0 & 0 & 2 & $\mathrm{CS}$ & Peq & umid & & & \\
\hline Sloanea guianensis (Aubl.) Benth. & AV & 0 & 0 & 2 & CS & Grd & Zoo & Cápsula & Bicolor & M \\
\hline $\begin{array}{l}\text { Sloanea monosperma Vell. } \\
\text { ERYTHROXYLACEAE }\end{array}$ & $\mathrm{AR}$ & 0 & 0 & 1 & $\mathrm{CS}$ & Grd & Zoo & Cápsula & Bicolor & A \\
\hline $\begin{array}{l}\text { Erythroxylum pelleterianum A.St.- } \\
\text { Hil. } \\
\text { ESCALLONIACEAE }\end{array}$ & AR & 6 & 0 & 2 & $\mathrm{CS}$ & Peq & Zoo & Drupa & Vermelho & A \\
\hline $\begin{array}{l}\text { Escallonia bifida Link. \& Otto } \\
\text { EUPHORBIACEAE }\end{array}$ & AV & 0 & 1 & 0 & CL & Méd & Ane & Cápsula & Marron & $\mathrm{V}$ \\
\hline $\begin{array}{l}\text { Alchornea glandulosa Poepp. \& } \\
\text { Endl. }\end{array}$ & AR & Flr & 0 & Flr & $\mathrm{P}$ & Grd & Zoo & Cápsula & Verde & A \\
\hline Alchornea sidifolia Müll.Arg. & $\mathrm{AR}$ & 0 & 4 & 28 & $\mathrm{P}$ & Méd & Zoo & Cápsula & Verde & A \\
\hline $\begin{array}{l}\text { Alchornea triplinervia (Spreng.) } \\
\text { Müll.Arg. }\end{array}$ & AR & 0 & 22 & 164 & CL & Grd & Zoo & Cápsula & Verde & $\mathrm{C}$ \\
\hline Croton celtidifolius Baill. & AR & 0 & 1 & 0 & $\mathrm{P}$ & Méd & Aut & Cápsula & Marron & $\mathrm{BL}$ \\
\hline Croton echinocarpus Müll.Arg. & AR & 0 & 4 & 0 & $\mathrm{P}$ & Méd & Aut & Cápsula & Bicolor & $\mathrm{BL}$ \\
\hline Croton floribundus Spreng. & AR & 24 & 0 & Flr & $\mathrm{P}$ & Grd & Aut & Cápsula & Marrom & $\mathrm{BL}$ \\
\hline Croton organensis Baill. & AR & 0 & 81 & 4 & $\mathrm{P}$ & Méd & Aut & Cápsula & Marrom & $\mathrm{BL}$ \\
\hline Croton salutaris Casar. & AR & 0 & 0 & 77 & $\mathrm{P}$ & Grd & Aut & Cápsula & Marrom & $\mathrm{BL}$ \\
\hline Manihot pilosa Pohl & $\mathrm{AB}$ & 0 & 0 & 2 & $\mathrm{CS}$ & Méd & Aut & Cápsula & Bicolor & BL \\
\hline Sapium glandulosum (L.) Morong & AR & 1 & 6 & 8 & CL & Grd & zoo & Cápsula & Verde & A \\
\hline $\begin{array}{l}\text { Sapium haematospermum } \\
\text { Müll.Arg. }\end{array}$ & $\mathrm{AR}$ & Flr & 0 & 0 & $\mathrm{P}$ & Méd & Zoo & Cápsula & Verde & A \\
\hline Sebastiania brasiliensis Spreng. & AR & 77 & 0 & 0 & $\mathrm{CS}$ & Peq & Aut & Cápsula & Castanho & $\mathrm{BL}$ \\
\hline $\begin{array}{l}\text { Sebastiania klotzschiana } \\
\text { (Müll.Arg.) Müll.Arg. }\end{array}$ & AR & 174 & 0 & 0 & CL & Méd & Aut & Cápsula & Castanho & $\mathrm{BL}$ \\
\hline $\begin{array}{l}\text { Tetrorchidium parvulum Müll.Arg. } \\
\text { FABACEAE }\end{array}$ & AR & 0 & 6 & 34 & $\mathrm{CS}$ & Méd & Aut & Cápsula & & $\mathrm{BL}$ \\
\hline Albizia polycephala (Benth.) Killip & AR & 0 & 0 & 8 & $\mathrm{CL}$ & Grd & Ane & Legume s.i & Creme & $\mathrm{V}$ \\
\hline $\begin{array}{l}\text { Anadenanthera colubrina (Vell.) } \\
\text { Brenan }\end{array}$ & AR & 139 & 1 & 0 & $\mathrm{P}$ & Grd & Ane & Legume s.i & $\begin{array}{l}\text { Marrom- } \\
\text { escuro }\end{array}$ & $\mathrm{V}$ \\
\hline Cassia ferruginea (Schrad.) Schrad. & $\mathrm{AR}$ & 35 & 0 & 0 & $\mathrm{CL}$ & Grd & Zoo & Legume s.i & $\begin{array}{l}\text { Marrom- } \\
\text { escuro }\end{array}$ & $\mathrm{A}$ e $\mathrm{M}$ \\
\hline Dalbergia villosa (Benth.) Benth. & AR & 0 & 0 & 21 & CL & Grd & Ane & Legume s.i & & $\mathrm{V}$ \\
\hline Erythrina falcata Benth. & AR & Flr & 0 & Flr & CL & Grd & Aut & Legume s.i & & $\mathrm{BL}$ \\
\hline Inga sessilis (Vell.) Mart. & AR & 0 & 1 & 4 & CL & Grd & Zoo & Legume c.i & Marrom & M \\
\hline Inga striata Benth. & AR & 0 & 0 & 2 & CL & Grd & Zoo & Legume c.i & Marrom & M \\
\hline Inga tenuis (Vell.) Mart. & $\mathrm{AR}$ & 0 & 0 & 26 & CL & Méd & Aut & Legume c.i & Castanho & \\
\hline Inga vulpina Mart. & AR & 12 & Flr & Flr & $\mathrm{CS}$ & Méd & Zoo & Legume c.i & Amarelo & M \\
\hline Machaerium brasiliense Vogel & AR & 2 & 0 & 0 & CL & Grd & Ane & Sâmara s.i & & V \\
\hline $\begin{array}{l}\text { Machaerium condensatum Kuhlm. } \\
\text { \& Hoehne }\end{array}$ & AR & 8 & 0 & 0 & $\mathrm{CS}$ & Méd & Ane & Sâmara s.i & & $\mathrm{V}$ \\
\hline Machaerium hirtum (Vell.) Stellfeld & AR & 13 & 0 & 0 & CL & Grd & Ane & Sâmara s.i & & $\mathrm{V}$ \\
\hline $\begin{array}{l}\text { Machaerium nyctitans (Vell.) } \\
\text { Benth. }\end{array}$ & AR & 0 & 0 & 1 & CL & Grd & Ane & Sâmara s.i & Castanho & $\mathrm{V}$ \\
\hline $\begin{array}{l}\text { Machaerium reticulatum (Peyr.) } \\
\text { Pers. }\end{array}$ & AR & 0 & 0 & 1 & CL & Méd & Ane & Sâmara s.i & Esverdeado & V \\
\hline Machaerium villosum Vogel & AR & 0 & 0 & 2 & CL & Grd & Ane & Sâmara s.i & & V \\
\hline
\end{tabular}

Continua ...

Ci. Fl., v. 20, n. 2, abr.-jun., 2010 
TABELA 1: Continuação ...

TABLE 1: Continued ...

\begin{tabular}{|c|c|c|c|c|c|c|c|c|c|c|}
\hline \multirow{2}{*}{ Família/Espécie } & \multirow{2}{*}{$\mathrm{Hab}$} & \multicolumn{3}{|c|}{ Áreas } & \multicolumn{3}{|c|}{ Guildas } & \multicolumn{2}{|c|}{ Fruto } & \multirow{2}{*}{$\mathrm{AD}$} \\
\hline & & I & II & III & Reg & Estr & Disp & Tipo & Cor & \\
\hline $\begin{array}{l}\text { Mimosa artemisiana } \text { Heringer \& } \\
\text { Paula }\end{array}$ & AR & Flr & 0 & Flr & $\mathrm{P}$ & Grd & Ane & Legume & Ferrugíneo & $\mathrm{V}$ \\
\hline Mimosa scabrela Benth. & AR & Flr & 7 & Flr & $\mathrm{P}$ & Grd & Ane & Craspédio & & V \\
\hline Ormosia fastigiata Tul. & AR & 0 & 0 & 7 & CL & Méd & Zoo & Legume & Bicolor & $\mathrm{V}$ \\
\hline $\begin{array}{l}\text { Senegalia polyphylla (DC.) Britton } \\
\text { \& Rose }\end{array}$ & $\mathrm{AR}$ & 2 & 0 & 0 & $\mathrm{P}$ & Grd & Ane & Legume s.i & & \\
\hline $\begin{array}{l}\text { Senna macranthera (Collad.) } \\
\text { H.S.Irwin \& Barneby }\end{array}$ & AR & 0 & 0 & 16 & $\mathrm{P}$ & Méd & Zoo & $\begin{array}{l}\text { Legume } \\
\text { s.d }\end{array}$ & Castanho & $\mathrm{BL}$ \\
\hline $\begin{array}{l}\text { Senna multijuga (L.C.Rich.) } \\
\text { H.S.Irwin \& Barneby }\end{array}$ & AR & 1 & 0 & 10 & $\mathrm{P}$ & Méd & Aut & Legume & Castanho & $\mathrm{BL}$ \\
\hline $\begin{array}{l}\text { Tachigali rugosa (Mart. ex Benth.) } \\
\text { Zarucchi \& Pipoly } \\
\text { HYPERICACEAE }\end{array}$ & $\mathrm{AB}$ & 0 & 1 & 26 & $\mathrm{CL}$ & Grd & Ane & Sâmara & Castanho & $\mathrm{BL}$ \\
\hline $\begin{array}{l}\text { Vismia brasiliensis Choisy } \\
\text { LAMIACEAE }\end{array}$ & AR & 0 & 0 & 1 & $\mathrm{CL}$ & Méd & Zoo & Baga & Amarelado & $\mathrm{A}$ e $\mathrm{M}$ \\
\hline Aegiphila fluminensis Vell. & AR & 0 & 2 & 6 & $\mathrm{P}$ & Méd & Zoo & Drupa & Vermelho & A \\
\hline Aegiphila obducta Vell. & $\mathrm{AB}$ & 3 & 2 & 0 & $\mathrm{P}$ & Méd & Zoo & Drupa & Alaranjado & A \\
\hline $\begin{array}{l}\text { Hyptidendron asperrimum (Epling) } \\
\text { Harley }\end{array}$ & $\mathrm{AB}$ & 0 & 0 & 28 & CL & Grd & Zoo & Drupa & & A \\
\hline $\begin{array}{l}\text { Vitex megapotamica } \text { (Spreng.) } \\
\text { Moldenke } \\
\text { LAURACEAE }\end{array}$ & $\mathrm{AB}$ & 39 & 0 & 0 & $\mathrm{CL}$ & Méd & Zoo & Drupa & Preto & A \\
\hline $\begin{array}{l}\text { Cinnamomum glaziovii (Mez) } \\
\text { Kosterm. }\end{array}$ & $\mathrm{AR}$ & 0 & 0 & Flr & $\mathrm{CS}$ & Grd & Zoo & Baga & Bicolor & A \\
\hline $\begin{array}{l}\text { Endlicheria paniculata (Spreng.) } \\
\text { J.F.Macbr. }\end{array}$ & AR & 0 & 0 & 5 & CS & Méd & Zoo & Baga & $\begin{array}{l}\text { Roxo- } \\
\text { vermelho }\end{array}$ & $\mathrm{A}$ e $\mathrm{M}$ \\
\hline Nectandra grandiflora Nees & AR & 0 & 6 & 1 & $\mathrm{CS}$ & Grd & Zoo & Baga & Preta & A \\
\hline Nectandra lanceolata Nees & AR & 7 & 0 & Flr & $\mathrm{CS}$ & Grd & Zoo & Drupa & Preto & M \\
\hline Nectandra oppositifolia Nees & AR & 0 & 0 & 15 & $\mathrm{CS}$ & Grd & Zoo & Baga & Roxo escuro & $\mathrm{A}$ e $\mathrm{M}$ \\
\hline Nectandra puberula (Schott) Nees & AR & 0 & 0 & 1 & CL & Grd & Zoo & Cápsula & Bicolor & A \\
\hline Ocotea aciphylla (Nees) Mez & AR & 0 & 1 & 11 & $\mathrm{CS}$ & Méd & Zoo & Baga & Preto & A \\
\hline Ocotea brachybotra (Meisn.) Mez & $\mathrm{AR}$ & 0 & 0 & 7 & $\mathrm{CS}$ & Grd & Zoo & Drupa & Roxo & $\mathrm{A}$ \\
\hline Ocotea corymbosa (Meisn.) Mez & AR & 0 & 5 & 39 & CL & Grd & Zoo & Baga & Bicolor & A \\
\hline Ocotea diospyrifolia (Meisn.) Mez & AR & 3 & 0 & 0 & CL & Grd & Zoo & Baga & Bicolor & M \\
\hline Ocotea indecora (Schott) $\mathrm{Mez}$ & AR & 2 & 1 & Flr & CL & Grd & Zoo & Baga & Multicolor & A \\
\hline Ocotea longifolia Kunth & AR & 0 & 0 & 6 & $\mathrm{CS}$ & Méd & Zoo & Baga & Bicolor & A \\
\hline Ocotea puberula (Rich.) Nees & AR & 1 & 0 & Flr & $\mathrm{CS}$ & Grd & Zoo & Baga & Preto & $\mathrm{A}$ e $\mathrm{M}$ \\
\hline Ocotea pulchella Mart. & AR & 4 & 0 & 0 & CL & Grd & Zoo & Baga & Roxo & $\mathrm{A}$ e $\mathrm{M}$ \\
\hline Ocotea silvestris Vattimo-Gil & AR & 0 & 1 & 0 & CL & Méd & Zoo & Baga & Amarelo & A \\
\hline Persea fulva Kopp & AR & 0 & 0 & Flr & CL & Grd & Zoo & Cápsula & Roxo & A \\
\hline Persea willdenovii Kosterm. & AR & 0 & 9 & 0 & CL & Grd & Zoo & Drupa & Azul & A \\
\hline $\begin{array}{l}\text { Persea venosa Nees } \\
\text { LECYTHIDACEAE }\end{array}$ & AR & 0 & 3 & 1 & CL & Grd & Zoo & Drupa & Preto & A \\
\hline $\begin{array}{l}\text { Couratari pyramidata (Vell.) } \\
\text { R.Knuth. } \\
\text { LYTHRACEAE }\end{array}$ & $\mathrm{AR}$ & 0 & 0 & 7 & $\mathrm{CS}$ & Méd & Aut & Pixídio & Marron & $\mathrm{BL}$ \\
\hline $\begin{array}{l}\text { Lafoensia pacari A.St.-Hil. } \\
\text { MALPIGHIACEAE }\end{array}$ & AR & 1 & 0 & 0 & $\mathrm{CS}$ & Grd & Ane & Cápsula & Marron & $\mathrm{V}$ \\
\hline Byrsonima myricifolia Griseb. & AR & 0 & 0 & 4 & CL & Grd & Zoo & Drupa & Vermelho & A \\
\hline $\begin{array}{l}\text { Byrsonima stipulacea A.Juss. } \\
\text { MALVACEAE }\end{array}$ & AR & 0 & 0 & 7 & $\mathrm{CL}$ & Grd & Zoo & Drupa & Amarelo & $\mathrm{M}$ \\
\hline $\begin{array}{l}\text { Pseudobombax grandiflorum (Cav.) } \\
\text { A.Robyns } \\
\text { MELASTOMATACEAE }\end{array}$ & $\mathrm{AB}$ & 0 & 0 & Flr & $\mathrm{CL}$ & Grd & Ane & Cápsula & Verde & $\mathrm{V}$ \\
\hline Huberia nettoana Brade & AR & 0 & 0 & 38 & CL & Grd & Ane & Velatídio & & $\mathrm{V}$ \\
\hline Leandra carassana (DC.) Cogn. & AR & 0 & 2 & 0 & $\mathrm{CS}$ & Peq & Zoo & Baga & & A \\
\hline Leandra melastomoides Raddi & $\mathrm{AB}$ & 0 & 3 & Flr & $\mathrm{CS}$ & Méd & Zoo & Baga & Preto & A \\
\hline Meriania claussenii Triana & $\mathrm{AB}$ & 0 & 0 & 1 & CL & Méd & Zoo & Baga & & A \\
\hline Miconia brunnea Mart. & AR & 0 & 0 & Flr & CS & Méd & Zoo & Baga & Preto & $\mathrm{A}$ \\
\hline
\end{tabular}


Pereira, I. M. et al.

TABELA 1: Continuação ...

TABLE 1: Continued ...

\begin{tabular}{|c|c|c|c|c|c|c|c|c|c|c|}
\hline \multirow{2}{*}{ Família/Espécie } & \multirow{2}{*}{$\mathrm{Hab}$} & \multicolumn{3}{|c|}{ Áreas } & \multicolumn{3}{|c|}{ Guildas } & \multicolumn{2}{|c|}{ Fruto } & \multirow{2}{*}{$\mathrm{AD}$} \\
\hline & & $\mathrm{I}$ & II & III & Reg & Estr & Disp & Tipo & Cor & \\
\hline Miconia budlejoides Triana & AV & 0 & 1 & 0 & $\mathrm{CL}$ & Méd & Zoo & Baga & & $\mathrm{A}$ \\
\hline Miconia chartacea Triana & AR & 0 & 1 & 12 & CL & Méd & Zoo & Baga & Preto & $\mathrm{A}$ \\
\hline Miconia cinerascens Miq. & AV & 0 & 1 & Flr & CL & Méd & Zoo & Baga & Preto & $\mathrm{A}$ \\
\hline $\begin{array}{l}\text { Miconia cinnamomifolia (DC.) } \\
\text { Naudin }\end{array}$ & AV & 0 & 16 & 18 & $\mathrm{P}$ & Grd & Zoo & Baga & Preto & $A$ e $M$ \\
\hline Miconia cubatanensis Hoehne & AR & 20 & 0 & 5 & $\mathrm{CL}$ & Méd & Zoo & Baga & Preto & $\mathrm{A}$ e $\mathrm{M}$ \\
\hline Miconia eichlerii Cogn. & AV & 0 & Flr & Flr & $\mathrm{CL}$ & Méd & Zoo & Baga & & A \\
\hline Miconia fasciculata Gardn. & AR & 0 & 3 & Flr & $\mathrm{CS}$ & Méd & Zoo & Baga & Preto & A \\
\hline Miconia latecrenata (DC.) Naudin & $\mathrm{AB}$ & 0 & 2 & 10 & $\mathrm{CL}$ & Méd & Zoo & Baga & Roxo & A \\
\hline Miconia minutiflora (Bonpl.) DC. & $\mathrm{AR}$ & 0 & 0 & Flr & $\mathrm{CL}$ & Méd & Zoo & Baga & Roxo & $\mathrm{A}$ \\
\hline Miconia prasina (Sw.) DC. & $\mathrm{AR}$ & Flr & Flr & 26 & $\mathrm{CS}$ & Méd & Zoo & Baga & Roxo & A \\
\hline Miconia pusilliflora (DC.) Triana & AV & Flr & 1 & 7 & CL & Méd & Zoo & Baga & Azul & $\mathrm{A}$ \\
\hline Miconia sellowiana Naudin & $\mathrm{AB}$ & 0 & 56 & 5 & $\mathrm{CL}$ & Méd & Zoo & Baga & Vermelho & $\mathrm{A}$ \\
\hline Miconia theaezans (Bonpl.) Cogn & $\mathrm{AB}$ & 0 & 39 & 0 & CL & Méd & Zoo & Baga & Preto & $\mathrm{A}$ \\
\hline Miconia valtherii Naudin & $\mathrm{AV}$ & 0 & 0 & 28 & $\mathrm{CL}$ & Méd & Zoo & Baga & & $\mathrm{A}$ \\
\hline Tibouchina arborea (Gardn.) Cogn. & $\mathrm{AB}$ & 0 & 72 & 8 & $\mathrm{CL}$ & Méd & Ane & Cápsula & & $\mathrm{V}$ \\
\hline $\begin{array}{l}\text { Tibouchina moricandiana (DC.) } \\
\text { Baill. }\end{array}$ & AR & Flr & 0 & Flr & $\mathrm{CL}$ & Méd & Ane & Cápsula & & $\mathrm{V}$ \\
\hline Tibouchina mutabilis Cogn. & $\mathrm{AB}$ & 0 & 1 & 1 & CL & Méd & Aut & Cápsula & & $\mathrm{V}$ \\
\hline Tibouchina pulchra Cogn. & AR & 0 & 5 & 106 & $\mathrm{CL}$ & Grd & Ane & Cápsula & & V \\
\hline $\begin{array}{l}\text { Tibouchina sellowiana (Cham.) } \\
\text { Cogn. }\end{array}$ & $\mathrm{AR}$ & 0 & 3 & Flr & $\mathrm{CL}$ & Méd & Ane & Cápsula & & $\mathrm{V}$ \\
\hline $\begin{array}{l}\text { Trembleya parviflora (D.Don) } \\
\text { Cogn. } \\
\text { MELIACEAE }\end{array}$ & AV & 0 & 2 & 0 & $\mathrm{P}$ & Méd & Zoo & Cápsula & Vermelho & $\mathrm{A}$ \\
\hline Cabralea canjerana (Vell.) Mart. & $\mathrm{AB}$ & 0 & 1 & 50 & $\mathrm{CS}$ & Grd & Zoo & Cápsula & Avermelhado & A \\
\hline Cedrela fissilis Vell. & AR & 0 & 1 & 3 & $\mathrm{CL}$ & Grd & Ane & Cápsula & Castanho & $\mathrm{V}$ \\
\hline Guarea kunthiana A.Juss. & AR & 0 & 0 & 1 & $\mathrm{CS}$ & Grd & Zoo & Cápsula & Bicolor & A e $M$ \\
\hline $\begin{array}{l}\text { Trichilia emarginata (Turcz.) } \\
\text { C.DC. }\end{array}$ & AR & 1 & 0 & 0 & $\mathrm{CS}$ & Méd & Zoo & Cápsula & Bicolor & A \\
\hline $\begin{array}{l}\text { Trichilia silvatica C.DC. } \\
\text { MONIMIACEAE }\end{array}$ & $\mathrm{AR}$ & 0 & 0 & 1 & $\mathrm{CL}$ & Grd & Zoo & Cápsula & Bicolor & A \\
\hline Mollinedia elegans Tul. & AR & 0 & 0 & 25 & $\mathrm{CS}$ & Méd & Zoo & Drupa & Preto & A \\
\hline Mollinedia engleriana Perkins & AR & 0 & 0 & 1 & $\mathrm{CS}$ & Méd & Zoo & Drupa & Preto & $\mathrm{A}$ \\
\hline Mollinedia fruticulosa Perkins & $\mathrm{AR}$ & 0 & Flr & 0 & CS & Peq & Zoo & Drupa & Preto & $\mathrm{A}$ \\
\hline $\begin{array}{l}\text { Mollinedia lamprophylla Perkins } \\
\text { MORACEAE }\end{array}$ & AR & 0 & 0 & Flr & $\mathrm{CS}$ & Méd & Zoo & Drupa & Preto & A \\
\hline Ficus luschnathiana (Miq.) Miq. & $\mathrm{AB}$ & 0 & 0 & 1 & $\mathrm{CS}$ & Grd & Zoo & Sicônio & Amarelado & $\mathrm{A}$ e $\mathrm{M}$ \\
\hline Ficus enormis (Mart.) Miq. & AR & 0 & 1 & 4 & $\mathrm{CS}$ & Grd & Zoo & Sicônio & & A \\
\hline $\begin{array}{l}\text { Sorocea bonplandii (Baill.) } \\
\text { W.Burger } \\
\text { MYRISTICACEAE }\end{array}$ & AR & Flr & Flr & 3 & $\mathrm{CS}$ & Méd & Zoo & Drupa & Bicolor & A e $M$ \\
\hline $\begin{array}{l}\text { Virola bicuhyba (Schott) Warb. } \\
\text { MYRSINACEAE }\end{array}$ & AR & 0 & 0 & Flr & $\mathrm{CL}$ & Grd & Zoo & Cápsula & Vermelho & $\mathrm{A}$ e $\mathrm{M}$ \\
\hline $\begin{array}{l}\text { Myrsine coriacea (Sw.) Roem. \& } \\
\text { Schult. }\end{array}$ & AR & 0 & 16 & 1 & $\mathrm{P}$ & Méd & Zoo & Drupa & Preto & A e $M$ \\
\hline Myrsine gardneriana A.DC. & $\mathrm{AR}$ & 58 & 22 & 7 & CL & Méd & Zoo & Drupa & Roxo & A \\
\hline Myrsine lineata (Mez) Imkhan. & AR & 0 & 2 & 0 & $\mathrm{CL}$ & Méd & Zoo & Drupa & & \\
\hline Myrsine umbellata Mart. & AR & 0 & 126 & 74 & CL & Méd & Zoo & Drupa & Vermelho & $\mathrm{A}$ e $\mathrm{M}$ \\
\hline $\begin{array}{l}\text { Myrsine villosissima Mart. } \\
\text { MYRTACEAE }\end{array}$ & AR & 0 & Flr & 0 & $\mathrm{CL}$ & Méd & Zoo & Drupa & Preto & A \\
\hline $\begin{array}{l}\text { Blepharocalyx salicifolius (Kunth) } \\
\text { O.Berg }\end{array}$ & AR & 13 & 0 & 2 & $\mathrm{CL}$ & Grd & Zoo & Baga & Vermelho & A \\
\hline Calyptranthes grandifolia O.Berg & AR & 1 & 0 & 0 & $\mathrm{CS}$ & Méd & Zoo & Baga & Roxo & A \\
\hline Calyptranthes widgreniana O.Berg & AR & 14 & 0 & Flr & $\mathrm{CS}$ & Grd & Zoo & Baga & Roxo-escuro & $\mathrm{A}$ \\
\hline $\begin{array}{l}\text { Campomanesia guaviroba (DC.) } \\
\text { Kiaersk. }\end{array}$ & $\mathrm{AR}$ & 0 & 0 & 1 & $\mathrm{CL}$ & Peq & Zoo & Baga & Amarelo & A \\
\hline $\begin{array}{l}\text { Campomanesia guazumifolia } \\
\text { (Cambess.) O.Berg }\end{array}$ & AR & 3 & 0 & 0 & $\mathrm{CL}$ & Méd & Zoo & Baga & Alaranjado & M \\
\hline
\end{tabular}

Continua $\ldots$

Ci. Fl., v. 20, n. 2, abr.-jun., 2010 
TABELA 1: Continuação ...

TABLE 1: Continued ...

\begin{tabular}{|c|c|c|c|c|c|c|c|c|c|c|}
\hline \multirow{2}{*}{ Família/Espécie } & \multirow{2}{*}{$\mathrm{Hab}$} & \multicolumn{3}{|c|}{ Áreas } & \multicolumn{3}{|c|}{ Guildas } & \multicolumn{2}{|c|}{ Fruto } & \multirow{2}{*}{$\mathrm{AD}$} \\
\hline & & I & II & III & Reg & Estr & Disp & Tipo & Cor & \\
\hline Campomanesia laurifolia Gardn. & AR & 7 & 0 & 0 & CL & Méd & Zoo & Baga & Amarelo & M \\
\hline Eugenia brasiliensis Lam. & $\mathrm{AR}$ & 0 & 0 & 1 & CS & Grd & Zoo & Baga & Preto & A \\
\hline Eugenia cerasiflora Miq. & AR & 1 & 0 & 8 & $\mathrm{CS}$ & Méd & Zoo & Baga & Vermelho & A \\
\hline Eugenia dodonaeifolia Cambess. & $\mathrm{AR}$ & 0 & 4 & 0 & CS & Grd & Zoo & Baga & Vermelho & M \\
\hline Eugenia involucrata DC. & $\mathrm{AR}$ & 0 & 0 & Flr & CL & Méd & Zoo & Baga & Preto & M \\
\hline Eugenia mansoi O.Berg & AR & 7 & 0 & 0 & $\mathrm{CL}$ & Méd & Zoo & Baga & Laranja & $\mathrm{A}$ \\
\hline $\begin{array}{l}\text { Gomidesia anacardiifolia (Gardn.) } \\
\text { O.Berg }\end{array}$ & $\mathrm{AR}$ & 0 & Flr & 0 & CL & Méd & Zoo & Baga & Vermelho & M \\
\hline Marlierea excoriata Mart. & $\mathrm{AR}$ & 1 & 0 & 0 & CS & Méd & Zoo & Baga & Preto & A \\
\hline $\begin{array}{l}\text { Marlierea racemosa (Vell.) } \\
\text { Kiaersk. }\end{array}$ & $\mathrm{AR}$ & 1 & 0 & 6 & CL & Méd & Zoo & Baga & Preto & A \\
\hline $\begin{array}{l}\text { Myrceugenia acutiflora (Kiaersk.) } \\
\text { D.Legrand \& Kausel }\end{array}$ & $\mathrm{AR}$ & 2 & 0 & 0 & CL & Méd & Zoo & Baga & Vermelho & A \\
\hline $\begin{array}{l}\text { Myrceugenia bracteosa (DC.) } \\
\text { D.Legrand \& Kausel }\end{array}$ & $\mathrm{AR}$ & 27 & 0 & 1 & CL & Méd & Zoo & Baga & Amarelo & A \\
\hline $\begin{array}{l}\text { Myrceugenia miersiana (Gardn.) } \\
\text { D.Legrand \& Kausel }\end{array}$ & $\mathrm{AR}$ & 0 & 0 & 7 & CS & Méd & Zoo & Baga & Preto & A \\
\hline $\begin{array}{l}\text { Myrcia diaphana }(\mathrm{O} . \mathrm{Berg}) \\
\text { N.Silveira }\end{array}$ & $\mathrm{AR}$ & 0 & Flr & 8 & CS & Méd & Zoo & Baga & Preto & A \\
\hline Myrcia eriopus DC. & AV & 0 & 0 & 25 & CS & Méd & Zoo & Baga & Vermelho & A \\
\hline Myrcia laruotteana Cambess. & $\mathrm{AB}$ & Flr & 0 & Flr & CL & Méd & Zoo & Baga & Preto & A \\
\hline Myrcia multiflora (Lam.) DC. & AV & 5 & 0 & 0 & $\mathrm{CS}$ & Grd & Zoo & Baga & Roxo & A \\
\hline Myrcia obovata (O.Berg) Nied. & $\mathrm{AR}$ & 0 & 0 & Flr & CL & Méd & Zoo & Baga & Vermelho & M \\
\hline Myrcia pulchra Kiaersk. & $\mathrm{AR}$ & 2 & 1 & 1 & $\mathrm{CS}$ & Méd & Zoo & Baga & Preto & A \\
\hline Myrcia splendens (Sw.) DC. & AR & 0 & 7 & 60 & CL & Méd & Zoo & Baga & Preto & A e $M$ \\
\hline $\begin{array}{l}\text { Myrciaria floribunda (H.West ex } \\
\text { Willd.) O.Berg }\end{array}$ & $\mathrm{AB}$ & 0 & 0 & Flr & CS & Méd & Zoo & Baga & Vermelho & M \\
\hline Myrciaria tenella (DC.) O.Berg & $\mathrm{AR}$ & 1 & 0 & Flr & $\mathrm{CS}$ & Méd & Zoo & Baga & Vermelho & A \\
\hline Plinia cauliflora (Mart.) Kausel & $\mathrm{AR}$ & 0 & 0 & Flr & CS & Grd & zoo & Baga & Preto & A \\
\hline Siphoneugena densiflora O.Berg & $\mathrm{AR}$ & 0 & 1 & 22 & CS & Méd & Zoo & Baga & Preto & A \\
\hline NYCTAGINACEAE & $\mathrm{AR}$ & & & & & & & & & \\
\hline $\begin{array}{l}\text { Guapira opposita (Vell.) Reitz } \\
\text { OCHNACEAE }\end{array}$ & AR & 0 & 16 & 30 & CS & Méd & Zoo & Drupa & Vermelho & A \\
\hline $\begin{array}{l}\text { Ouratea parviflora (DC.) Baill. } \\
\text { PERACEAE }\end{array}$ & $\mathrm{AB}$ & 3 & 0 & 4 & $\mathrm{CS}$ & Méd & Zoo & Drupa & Bicolor & A \\
\hline $\begin{array}{l}\text { Pera glabrata (Schott) Poepp. } \\
\text { PICRAMNIACEAE }\end{array}$ & $\mathrm{AR}$ & 0 & 0 & 12 & CL & Grd & Zoo & Cápsula & Bicolor & A \\
\hline $\begin{array}{l}\text { Picramnia glazioviana Engl. } \\
\text { PIPERACEAE }\end{array}$ & $\mathrm{AR}$ & 0 & Flr & Flr & CS & Méd & Zoo & Baga & Vermelho & A \\
\hline Piper amalago L. & AR & 0 & 1 & 0 & $\mathrm{CS}$ & Méd & Zoo & Drupa & Verde & $\mathrm{M}$ \\
\hline Piper cernuum Vell. & $\mathrm{AB}$ & 0 & 0 & 1 & $\mathrm{CS}$ & Peq & Zoo & Drupa & Verde & M \\
\hline Piper crassinervium Kunth & $\mathrm{AB}$ & 0 & 0 & Flr & $\mathrm{CS}$ & Peq & Zoo & Drupa & Verde & M \\
\hline $\begin{array}{l}\text { Piper dilatatum } \text { L.C.Rich } \\
\text { PODOCARPACEAE }\end{array}$ & $\mathrm{AB}$ & 0 & 0 & 1 & $\mathrm{CS}$ & Peq & Zoo & Drupa & Verde & M \\
\hline $\begin{array}{l}\text { Podocarpus lambertii Klotzsch } \\
\text { PROTEACEAE }\end{array}$ & $\mathrm{AB}$ & 0 & 1 & Flr & $\mathrm{P}$ & Méd & Zoo & Baga & Roxo-escuro & A \\
\hline Euplassa legalis (Vell.) I.M.Johnst. & $\mathrm{AB}$ & 0 & 0 & 2 & CL & Grd & Zoo & Noz s.d & Amarelado & A \\
\hline $\begin{array}{l}\text { Euplassa organensis (Gardn.) } \\
\text { I.M.Johnst. }\end{array}$ & $\mathrm{AR}$ & 0 & 0 & 16 & CL & Grd & Zoo & Noz s.d & Amarelado & A \\
\hline Roupala montana Aubl. & $\mathrm{AR}$ & 0 & 1 & Flr & CL & Grd & Ane & $\begin{array}{l}\text { Folículo } \\
\text { s.d }\end{array}$ & Castanho & $\mathrm{V}$ \\
\hline $\begin{array}{l}\text { QUIINACEAE } \\
\text { Quiina glaziovii Engl. } \\
\text { ROSACEAE }\end{array}$ & $\mathrm{AR}$ & 0 & Flr & 0 & CS & Méd & Zoo & Baga & Laranja & M \\
\hline $\begin{array}{l}\text { Prunus myrtifolia (L.) Urb. } \\
\text { RUBIACEAE }\end{array}$ & $\mathrm{AR}$ & 6 & 18 & 26 & CS & Méd & Zoo & Drupa & Roxo-escuro & $\mathrm{A}$ e $\mathrm{M}$ \\
\hline $\begin{array}{l}\text { Amaioua intermedia Mart. ex } \\
\text { Schult. \& Schult.f. }\end{array}$ & $\mathrm{AR}$ & 0 & 2 & 15 & CS & Méd & Zoo & Baga & Roxo & A \\
\hline Bathysa australis (A.St.-Hil.) & AV & 0 & 0 & 6 & $\mathrm{CS}$ & Méd & Ane & Cápsula & & V \\
\hline
\end{tabular}


Pereira, I. M. et al.

TABELA 1: Continuação ...

TABLE 1: Continued ...

\begin{tabular}{|c|c|c|c|c|c|c|c|c|c|c|}
\hline \multirow{2}{*}{ Família/Espécie } & \multirow{2}{*}{$\mathrm{Hab}$} & \multicolumn{3}{|c|}{ Áreas } & \multicolumn{3}{|c|}{ Guildas } & \multicolumn{2}{|c|}{ Fruto } & \multirow{2}{*}{$\mathrm{AD}$} \\
\hline & & I & II & III & Reg & Estr & Disp & Tipo & Cor & \\
\hline \multicolumn{11}{|l|}{ Benth. \& Hook.f. } \\
\hline Chomelia brasiliana A.Rich. & $\mathrm{AR}$ & 42 & 0 & 0 & $\mathrm{CS}$ & Grd & Zoo & Baga & Preto & \\
\hline Cordiera concolor (Cham.) Kuntze & $\mathrm{AR}$ & 2 & 0 & 0 & $\mathrm{CL}$ & Peq & Zoo & Baga & Preto & A \\
\hline $\begin{array}{l}\text { Coutarea hexandra (Jacq.) } \\
\text { K.Schum. }\end{array}$ & $\mathrm{AR}$ & 2 & 0 & 0 & CS & Méd & Ane & Cápsula & Verde-escuro & $\mathrm{V}$ \\
\hline Hillia parasitica Jacq. & $\mathrm{AB}$ & 0 & 0 & Flr & CS & Peq & Ane & & & V \\
\hline Psychotria suterella Müll.Arg. & $\mathrm{AR}$ & 0 & 1 & 2 & $\mathrm{CS}$ & Peq & Zoo & Drupa & Azul & $\mathrm{A}$ e $\mathrm{M}$ \\
\hline Psychotria vellosiana Benth. & $\mathrm{AB}$ & 0 & 51 & 213 & $\mathrm{CL}$ & Peq & Zoo & Drupa & Preto & \\
\hline $\begin{array}{l}\text { Rudgea jasminoides (Cham.) } \\
\text { Müll.Arg. }\end{array}$ & $\mathrm{AB}$ & 0 & 0 & 2 & CS & Méd & Zoo & Drupa & Vermelho & A \\
\hline $\begin{array}{l}\text { Rudgea recurva Müll.Arg. } \\
\text { RUTACEAE }\end{array}$ & $\mathrm{AB}$ & 0 & 0 & 1 & $\mathrm{CS}$ & Méd & Zoo & Drupa & Branco & A \\
\hline Esenbeckia grandiflora Mart. & $\mathrm{AR}$ & 0 & 2 & 10 & $\mathrm{CS}$ & Peq & Aut & Cápsula & Bicolor & $\mathrm{BA}$ \\
\hline Pilocarpus pauciflorus A.St.-Hil. & $\mathrm{AB}$ & 0 & 0 & Flr & $\mathrm{CS}$ & Peq & Aut & Cápsula & Amarelado & $\mathrm{BA}$ \\
\hline $\begin{array}{l}\text { Zanthoxylum rhoifolium Lam. } \\
\text { SABIACEAE }\end{array}$ & $\mathrm{AR}$ & 0 & 0 & 6 & $\mathrm{CL}$ & Grd & Zoo & Cápsula & Vinaceo & A \\
\hline Meliosma brasiliensis Urb. & $\mathrm{AR}$ & 0 & 6 & 2 & $\mathrm{CS}$ & Méd & Zoo & Drupa & & \\
\hline Meliosma sellowii Urb. & AR & 0 & 0 & Flr & $\mathrm{CS}$ & Méd & Zoo & Drupa & Verde & A \\
\hline $\begin{array}{l}\text { Meliosma sinuata Urb. } \\
\text { SALICACEAE }\end{array}$ & $\mathrm{AR}$ & 0 & 0 & 3 & $\mathrm{CS}$ & Méd & Zoo & Drupa & & \\
\hline Casearia arborea (L.C.Rich.) Urb. & $\mathrm{AR}$ & 0 & 0 & 86 & CL & Grd & Zoo & Cápsula & Roxo & A \\
\hline Casearia decandra Jacq. & $\mathrm{AR}$ & 0 & 0 & 26 & CS & Méd & Zoo & Cápsula & Laranja & $\mathrm{A}$ e $\mathrm{M}$ \\
\hline Casearia mariquitensis Kunth & $\mathrm{AB}$ & 0 & 0 & Flr & $\mathrm{CS}$ & Méd & Zoo & Cápsula & Bicolor & A \\
\hline Casearia obliqua Spreng. & $\mathrm{AR}$ & 0 & 0 & 34 & $\mathrm{CL}$ & Grd & Zoo & Cápsula & Bicolor & A \\
\hline Casearia pauciflora Cambess. & $\mathrm{AR}$ & 0 & 0 & 22 & $\mathrm{CL}$ & Grd & Zoo & Cápsula & Verde & M \\
\hline Casearia rupestris Eichler & AR & 0 & 2 & 0 & $\mathrm{CL}$ & Peq & Zoo & Cápsula & Verde escuro & A \\
\hline Casearia sylvestris $\mathrm{Sw}$ & $\mathrm{AR}$ & 4 & 0 & 0 & $\mathrm{P}$ & Méd & Zoo & Cápsula & Verde escuro & A \\
\hline Xylosma prockia (Turcz.) Turcz. & $\mathrm{AR}$ & 10 & Flr & 0 & $\mathrm{CL}$ & Méd & Zoo & Baga & Preto & A \\
\hline $\begin{array}{l}\text { Xylosma venosa N.E.Brown } \\
\text { SAPINDACEAE }\end{array}$ & $\mathrm{AR}$ & 0 & 0 & 1 & $\mathrm{CL}$ & Méd & Zoo & Baga & Preto & A \\
\hline $\begin{array}{l}\text { Allophylus edulis (A.St.-Hil., } \\
\text { Cambess. \& A.Juss.) Radlk. }\end{array}$ & $\mathrm{AR}$ & 13 & 0 & 3 & $\mathrm{CS}$ & Méd & Zoo & Drupa & Vermelho & $\mathrm{A}$ e $\mathrm{M}$ \\
\hline $\begin{array}{l}\text { Allophylus guaraniticus (A.St.-Hil.) } \\
\text { Radlk. }\end{array}$ & $\mathrm{AR}$ & 1 & 0 & 0 & $\mathrm{CS}$ & Méd & Zoo & Drupa & Multicolor & A \\
\hline Cupania concolor Radlk. & $\mathrm{AR}$ & 0 & 15 & 0 & $\mathrm{CL}$ & Méd & Ane & Cápsula & Multicolor & $\mathrm{A}$ \\
\hline $\begin{array}{l}\text { Cupania ludowigii Somner \& } \\
\text { Ferruci }\end{array}$ & $\mathrm{AR}$ & 1 & 0 & Flr & CS & Méd & Zoo & Cápsula & Multicolor & A \\
\hline Cupania vernalis Cambess. & $\mathrm{AR}$ & 22 & 1 & Flr & $\mathrm{CL}$ & Méd & Zoo & Cápsula & Multicolor & $\mathrm{A}$ \\
\hline Cupania zanthoxyloides Cambess. & $\mathrm{AB}$ & 0 & 0 & Flr & $\mathrm{CL}$ & Grd & Zoo & Cápsula & Marrom & A \\
\hline Matayba elaeagnoides Radlk. & $\mathrm{AR}$ & 108 & 0 & Flr & $\mathrm{CL}$ & Grd & Zoo & Cápsula & Avermelhado & A \\
\hline $\begin{array}{l}\text { Matayba juglandifolia (Cambess.) } \\
\text { Radlk. } \\
\text { SAPOTACEAE }\end{array}$ & $\mathrm{AR}$ & 0 & 0 & 15 & $\mathrm{CL}$ & Méd & Ane & Cápsula & Multicolor & A \\
\hline $\begin{array}{l}\text { Chrysophyllum gonocarpum (Mart. } \\
\text { \& Eichler) Engl. }\end{array}$ & $\mathrm{AR}$ & 0 & 0 & 1 & $\mathrm{CL}$ & Grd & Zoo & Drupa & Amarelo & $\mathrm{A}$ e $\mathrm{M}$ \\
\hline $\begin{array}{l}\text { Pouteria caimito (Ruiz \& Pav.) } \\
\text { Radlk. }\end{array}$ & $\mathrm{AR}$ & 0 & 0 & 3 & $\mathrm{CL}$ & Grd & Zoo & Baga & Amarelo & M \\
\hline $\begin{array}{l}\text { Pouteria guianensis Aubl. } \\
\text { SOLANACEAE }\end{array}$ & $\mathrm{AR}$ & 0 & 0 & Flr & $\mathrm{CL}$ & Grd & Zoo & Baga & Laranja & M \\
\hline $\begin{array}{l}\text { Brugmansia suaveolens (Humb. \& } \\
\text { Bonpl. ex Willd.) Bercht. \& J.Presl. }\end{array}$ & $\mathrm{AR}$ & 0 & 0 & Flr & $\mathrm{CL}$ & Peq & Aut & Baga & & \\
\hline Cestrum corymbosum Schltdl. & $\mathrm{AB}$ & 0 & 0 & Flr & $\mathrm{P}$ & Peq & Zoo & Baga & & $\mathrm{A}$ \\
\hline Cestrum schlechtendalii G.Don & $\mathrm{AB}$ & 0 & 1 & Flr & $\mathrm{CS}$ & Méd & Zoo & Baga & Preto & $\mathrm{A}$ \\
\hline Dyssochroma viridiflora Miers & $\mathrm{AB}$ & 0 & 0 & 1 & CS & Peq & Zoo & Baga & Verde & M \\
\hline Solanum bullatum Vell. & $\mathrm{AB}$ & 0 & 0 & 3 & $\mathrm{CL}$ & Méd & Zoo & Baga & Verde & A \\
\hline Solanum cinnamomeum Sendtn. & $\mathrm{AB}$ & 0 & 1 & 21 & $\mathrm{CL}$ & Méd & Zoo & Baga & Branco & A \\
\hline Solanum diploconos (Mart.) Bohs & $\mathrm{AR}$ & 0 & Flr & 0 & $\mathrm{CL}$ & Méd & Zoo & Baga & Preto & $\mathrm{A}$ \\
\hline Solanum granuloso-leprosum Dunal & $\mathrm{AB}$ & Flr & Flr & Flr & $\mathrm{P}$ & Méd & Zoo & Baga & Verde & $\mathrm{A}$ e $\mathrm{M}$ \\
\hline
\end{tabular}

Continua ... 
TABELA 1: Continuação ...

TABLE 1: Continued ...

\begin{tabular}{|c|c|c|c|c|c|c|c|c|c|c|}
\hline \multirow{2}{*}{ Família/Espécie } & \multirow{2}{*}{$\mathrm{Hab}$} & \multicolumn{3}{|c|}{ Áreas } & \multicolumn{3}{|c|}{ Guildas } & \multicolumn{2}{|c|}{ Fruto } & \multirow{2}{*}{$\mathrm{AD}$} \\
\hline & & $\mathrm{I}$ & II & III & Reg & Estr & Disp & Tipo & Cor & \\
\hline Solanum lepidotum Dunal & AR & 2 & 5 & Flr & $\mathrm{CL}$ & Méd & Zoo & Baga & Preto & A \\
\hline Solanum leucodendron Sendtn. & AR & 0 & 0 & Flr & CL & Grd & Zoo & Baga & Preto & $\mathrm{A}$ \\
\hline Solanum pseudoquina A.St.-Hil. & AR & 0 & 4 & 3 & $\mathrm{CL}$ & Peq & Zoo & Baga & Amarelado & $\mathrm{A}$ \\
\hline $\begin{array}{l}\text { Solanum swartzianum Roem. \& } \\
\text { Schult. } \\
\text { STYRACACEAE }\end{array}$ & AR & 0 & 1 & 12 & $\mathrm{CL}$ & Méd & Zoo & Baga & Arroxeado & M \\
\hline $\begin{array}{l}\text { Styrax maninul B.Walln. } \\
\text { SYMPLOCACEAE }\end{array}$ & $\mathrm{AR}$ & 0 & 0 & 4 & CL & Grd & Zoo & Brupa & Vermelho & A \\
\hline Symplocos celastrinea Mart. & AR & 122 & 1 & 3 & CL & Grd & Zoo & Drupa & Roxo & $\mathrm{A}$ \\
\hline $\begin{array}{l}\text { Symplocos insignis Brand } \\
\text { THEACEAE }\end{array}$ & $\mathrm{AB}$ & 0 & Flr & 1 & $\mathrm{CL}$ & Méd & Zoo & Drupa & & A \\
\hline $\begin{array}{l}\text { Laplacea fruticosa (Schrad.) } \\
\text { Kobuski } \\
\text { THYMELAEACEAE }\end{array}$ & $\mathrm{AR}$ & 0 & 3 & 20 & $\mathrm{CL}$ & Méd & Zoo & Cápsula & $\begin{array}{l}\text { Castanho- } \\
\text { pardacento }\end{array}$ & $\mathrm{V}$ \\
\hline Daphnopsis coriacea Taub. & AR & 4 & 0 & 0 & CL & Méd & Zoo & Núcula & Verde & A \\
\hline $\begin{array}{l}\text { Daphnopsis fasciculata (Meisn.) } \\
\text { Nevling }\end{array}$ & AR & 16 & 0 & 12 & $\mathrm{CL}$ & Méd & Zoo & Núcula & Laranja & A \\
\hline Daphnopsis martii Meisn. & AR & 0 & 3 & 0 & $\mathrm{CS}$ & Méd & Zoo & Núcula & Laranja & A \\
\hline $\begin{array}{l}\text { Daphnopsis sellowiana Taub. } \\
\text { URTICACEAE }\end{array}$ & AR & 15 & 0 & 0 & $\mathrm{CS}$ & Méd & Zoo & Núcula & Laranja & $\mathrm{A}$ \\
\hline Cecropia glaziovii Snethl. & AR & 0 & 0 & 2 & $\mathrm{P}$ & Méd & Zoo & Sincárrpico & Roxo & $\mathrm{A}$ e $\mathrm{M}$ \\
\hline Cecropia hololeuca Miq. & AR & 0 & 0 & 12 & $\mathrm{P}$ & Grd & Zoo & Sincárrpico & Preto & $\mathrm{A}$ e $\mathrm{M}$ \\
\hline $\begin{array}{l}\text { Coussapoa microcarpa (Schott) } \\
\text { Rizz. } \\
\text { VERBENACEAE }\end{array}$ & $\mathrm{AR}$ & 0 & 0 & Flr & $\mathrm{CL}$ & Méd & Zoo & Sincárrpico & Amarelo & M \\
\hline $\begin{array}{l}\text { Duranta vestita Cham. } \\
\text { VOCHYSIACEAE }\end{array}$ & $\mathrm{AB}$ & 30 & 0 & 0 & $\mathrm{CL}$ & Peq & Zoo & Drupa & Bege & $\mathrm{A}$ \\
\hline Vochysia magnifica Warm. & $\mathrm{AB}$ & 0 & 0 & 183 & CL & Grd & Ane & Cápsula & Verde & $\mathrm{V}$ \\
\hline $\begin{array}{l}\text { Vochysia tucanorum Mart. } \\
\text { WINTERACEAE }\end{array}$ & $\mathrm{AB}$ & 0 & 1 & 0 & CL & Méd & Ane & Cápsula & Verde & $\mathrm{V}$ \\
\hline Drimys brasiliensis Miers & AR & 0 & 1 & 4 & $\mathrm{CL}$ & Méd & Zoo & Baga & Roxo & $\mathrm{A}$ \\
\hline
\end{tabular}

Em que: Hab. = hábito (AR: árvore, AB: arbusto e AV: arvoreta); I: mata ciliar de aluvião; II: mata ciliar de encosta em regeneração e III: mata ciliar de encosta conservada; $R$ eg = guildas de regeneração $(\mathrm{P}=$ pioneira, $\mathrm{CL}=\mathrm{clímax}$ exigente de luz e CS = clímax tolerante à sombra); Estr = guildas de estratificação (Peq: pequena, Med: média e Grd: grande); Disp = guildas de dispersão (Ane: anecórica, Zoo: zoocórica e auto: autocórica); AD = agente dispersor (A = aves, $\mathrm{A}$ e $\mathrm{M}=$ aves, mamíferos, $\mathrm{M}=$ mamíferos, $\mathrm{V}=$ vento, $\mathrm{BR}=$ barocoria e $\mathrm{BL}=$ balística). 


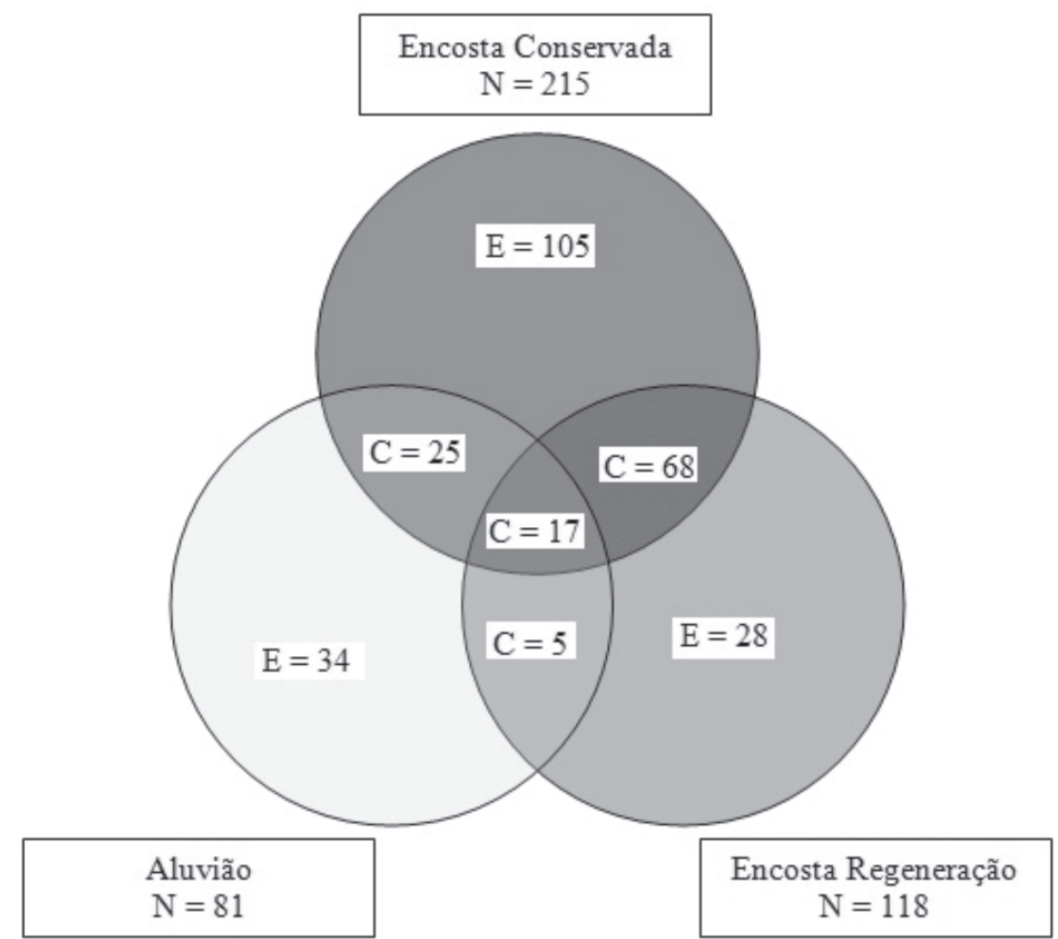

FIGURA 1: Diagrama de Venn extraído da composição florística das três áreas de mata ciliar inventariadas em Bocaina de Minas, MG mostrando o número de espécies compartilhadas. Em que: E= espécies exclusivas; $\mathrm{C}=$ espécies comuns entre as áreas; $\mathrm{N}=$ número total de espécies registradas na área.

FIGURE 1: Diagram of Venn extracted from the floristic composition of the three areas of ciliary forest inventoried in Bocaina of Minas, MG showing the number of shared species. In that: And = exclusive species; $\mathrm{C}=$ common species; $\mathrm{N}=$ total number of species registered in the area.

O diagrama de Venn (Figura 1) mostra claramente que uma pequena porcentagem, 5,59\% ou 17espécies, tem ocorrência comum nos três habitats. Verifica-se, ainda, que 27,38\% (68) das espécies registradas ocorrem exclusivamente nas duas áreas de encosta. Por outro lado, as menores proporções de espécies compartilhadas com exclusividade foram registradas entre as áreas de aluvião e encosta em regeneração, 2,82\% (cinco espécies) e conservada, 10\% (25 espécies), evidenciando que a área de aluvião possui composição de espécies diferentes das áreas de encosta. As áreas de mata ciliar situadas nos solos aluvionais são sítios de alta seletividade, pois poucas espécies estão adaptadas às condições de alagamento temporário desses ambientes. Tais informações devem nortear as ações que visam recompor a vegetação das matas ciliares das cabeceiras do rio Grande.

$\mathrm{Na}$ Figura 2, verifica-se que as proporções de espécies e indivíduos clímax exigentes de luz variaram pouco entre as três áreas. Os menores valores para espécies e indivíduos, respectivamente, foram registrados na encosta em regeneração
(46,61\% e 58,75\%), e os maiores valores na área de aluvião $(50,62 \%$ e $66,92 \%$ respectivamente). Constata-se também, nessa mesma figura, que as espécies tolerantes à sombra ocorreram em maiores proporções de espécies e indivíduos na encosta conservada $(40,93 \%$ e $27,85 \%$ respectivamente $)$ e em menores proporções de espécies na área de aluvião $(33,33 \%)$ e indivíduos na encosta em regeneração (14,60\%). Já as pioneiras ocorreram em maiores proporções de espécies $(18,64 \%)$ e indivíduos $(26,64 \%)$, na área de encosta em regeneração. Por outro lado, as menores proporções foram verificadas na encosta conservada $(11,16 \%$ e $9,99 \%$ respectivamente).

Com base nos resultados apresentados, podese inferir que, nas formações em estágios mais iniciais de sucessão ecológica, $66 \%$ das espécies e $83 \%$ dos indivíduos amostrados pertencem às espécies que dependem de luz durante todo seu ciclo de vida ou na fase inicial, evidenciando que, no planejamento das propostas de recomposição da vegetação das matas ciliares das cabeceiras do rio Grande, em torno de $66 \%$ das espécies devem ser de pioneiras e clímax exigente de luz, e que a proporção de mudas das 


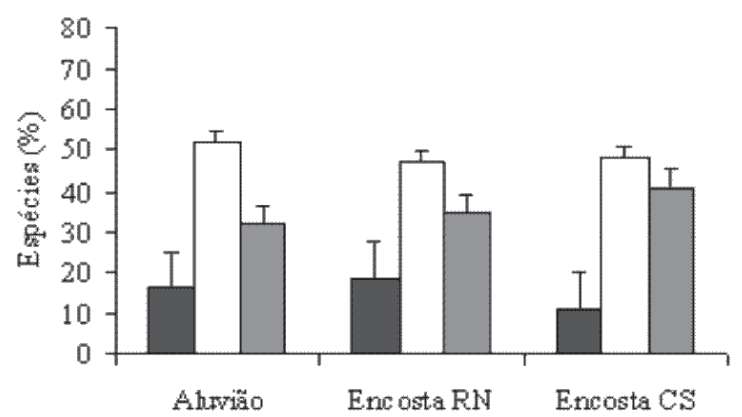

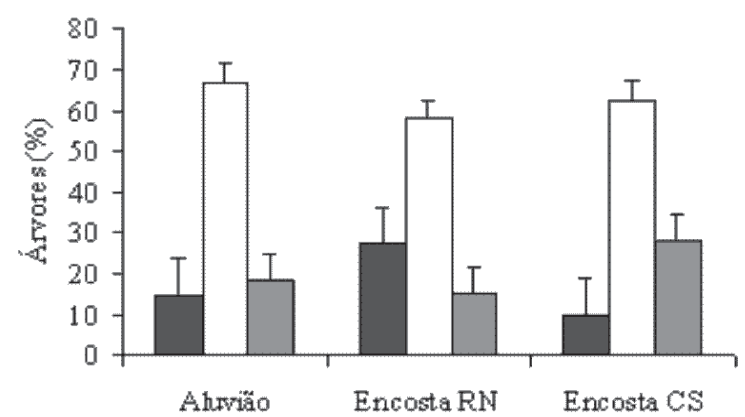

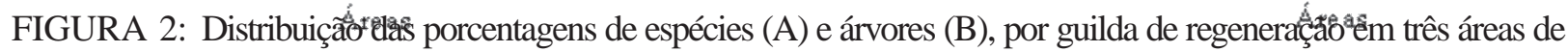
mata ciliar em Bocaina de Minas, MG. Em que: P: pioneira, CL: clímax exigente de luz, e CS: clímax tolerante à sombra.

FIGURE 2: Distribution of the percentage of species (A) and trees (B), for regeneration guilds in three areas of ciliary forest in Bocaina of Minas, MG. In that: P: pioneer, CL: climax demanding of light, and CS: climax tolerant to the shade.

espécies pertencentes a esses grupos de plantas seja de, no mínimo, $83 \%$ do total de mudas utilizadas. De acordo com Gómez-Pompa (1971), as espécies apresentam uma ampla variedade de respostas, havendo um gradiente de tolerância à luz nas diferentes fases do desenvolvimento. Para Rodrigues e Gandolfi (1996), as espécies de cada grupo sucessional apresentam características biológicas distintas, tais como dependência de diferentes intensidades de luz, velocidade de crescimento e duração do ciclo de vida. Assim, exigem diferentes condições ambientais para crescer, sobreviver e se reproduzir. Para Swaine e Whithmore (1988), as espécies pioneiras se estabelecem após perturbações que expõem o solo à luz. Já as espécies clímax exigente de luz também apresentam esse comportamento, porém, têm um ciclo de vida maior do que as pioneiras, tornando-se grandes árvores emergentes na floresta.

Constatou-se também neste estudo, que as espécies clímax tolerantes à sombra aumentaram sua importância em direção à maior maturidade das florestas, enquanto as espécies pioneiras apresentaram baixa abundância de indivíduos na área de encosta conservada, sendo estas restritas às clareiras e bordas com o rio e pastagem. Verificouse, ainda, que o número de espécies pioneiras registradas foi decrescente da área de floresta em estágio mais inicial de sucessão (encosta em regeneração) para a mais madura (encosta conservada). Esse padrão é comum ao citado na literatura (KLEIN, 1980; PESSOA et al., 1997; TABARELLI e MANTOVANI, 1999). Segundo os mesmos autores, esses resultados comprovam que o período de restabelecimento de uma floresta após distúrbio depende não apenas do tempo, mas também da intensidade do distúrbio e das condições do ambiente.

Analisando-se as três áreas isoladamente, pode-se inferir que a encosta em regeneração encontra-se em uma fase seral de inicial a intermediária, enquanto a encosta conservada apresentou características de uma floresta mais madura.

Na Figura 3, observa-se que as proporções de espécies com síndrome zoocórica variaram de 68,7 a 75,3\%, para as áreas encosta em regeneração e aluvião respectivamente (Figura 3A). Esses resultados evidenciam a importância das espécies zoocóricas nas matas ciliares das cabeceiras do rio Grande. O uso de espécies florestais que têm seus propágulos disseminados por animais nos programas de recomposição de mata ciliar é uma forma de promover um incremento da biodiversidade nas áreas a serem recuperadas, pois, no momento da frutificação, várias espécies de animais passarão a frequentar essas áreas em busca de alimento. Nesse processo, poderão ainda promover a troca de propágulos entre a área em recuperação e os fragmentos circunvizinhos e, consequentemente, realizar também a diversidade genética das populações de plantas entre os fragmentos. Oliveira Filho (1994) salienta a importância de considerar, na seleção das espécies que irão compor os plantios, o potencial destas em relação à atração da fauna pelo fornecimento de abrigo e de alimento. 
A

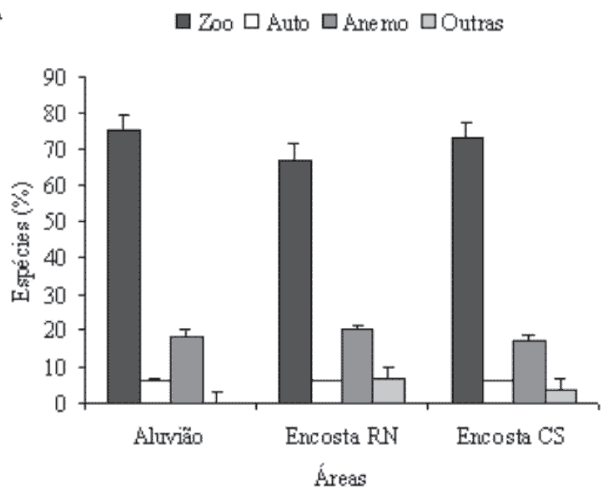

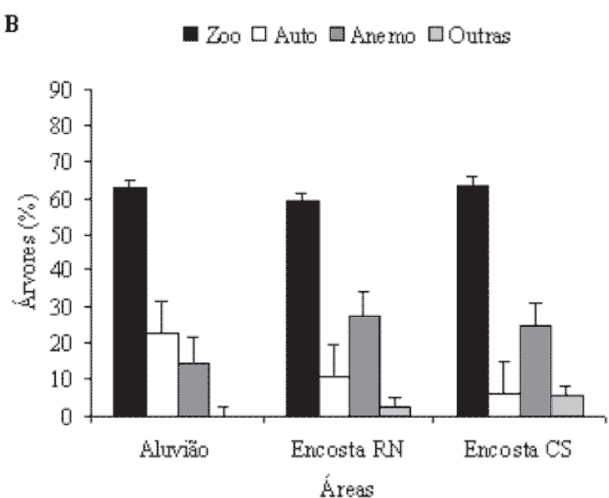

FIGURA 3: Distribuição das porcentagens de espécies (A) e árvores (B), por guilda de dispersão em três áreas de mata ciliar em Bocaina de Minas, MG. Em que Ane: anemocoria, Aut: autocoria, Zoo: zoocoria e Outras: outro tipo de dispersão.

FIGURE 3: Distribution of the percentage of species (A) and trees (B), for dispersion guilds in three areas of ciliary forest in Bocaina of Minas, MG. In that Ane: anemochory, Aut: autochory, Zoo: zoochory and Other: other dispersion type.

As espécies anemocóricas ocorreram em maiores proporções na encosta em regeneração (20,9\%), enquanto as menores proporções foram registradas na encosta conservada (17,7\%) (Figura 3A). As proporções de espécies autocóricas variaram pouco entre as áreas, com ocorrência em torno de $6 \%$.

As maiores proporções de indivíduos de espécies zoocóricas foram observadas na área de aluvião, $67 \%$, e as menores na encosta em regeneração, 58,3\% (Figura 3B). Quanto à categoria anemocórica, verifica-se que as maiores proporções foram encontradas na encosta em regeneração, $28,4 \%$ (Figura 3B). Os indivíduos de espécies autocóricas predominaram na área de aluvião, 22,9\%, e ocorreram em menores proporções na encosta conservada, 6,7\% (Figura 3B).

As altas proporções de espécies que têm suas fontes de propágulos disseminadas por animais (zoocóricas) registradas nas matas ciliares de Bocaina de Minas (68 a 75\%) encontram-se dentro dos limites descritos na literatura para outras florestas tropicais úmidas (GENTRY, 1982; HOWE e SMOLLWOOD, 1982; MORELLATO e LEITÃO FILHO, 1992).

Estima-se que 60 a 90\% das espécies arbóreas apresentem adaptações a esse tipo de dispersão, que aumenta sua importância quanto mais úmida for a floresta (HOWE e SMALLWOOD, 1982). Na dispersão por animal, há desde pequenos roedores e aves que, ao se alimentarem de frutos e sementes, fazem a dispersão por meio de suas sobras, até mamíferos e pássaros maiores que ingerem e defecam as sementes intactas, realizando sua dispersão (REIS, 1995). De acordo com Carmo e Morellato (2001), a proporção de espécies zoocóricas em área de mata ciliar disseminadas por animais é de $75 \%$. Já Martins et al., (1995) avaliaram a síndrome de dispersão de 520 espécies de Mata Atlântica e constataram que $75 \%$ delas tinham seus diásporos dispersos por animais. Esses valores são semelhantes aos obtidos para as matas ciliares das cabeceiras do rio Grande.

As proporções de espécies anemocóricas encontradas em Bocaina de Minas podem ser consideradas intermediárias $(17,7 \%$ a $20,9 \%)$, sendo inferiores próximos ou inferiores às obtidas por Morellato e Leitão Filho (1992) para florestas estacionais semidecíduas (20 a $28 \%$ ), iguais às obtidas por Martins et al., (1995) para mata atlântica (18\%) e por Carmo e Morellato (2001) para áreas de mata ciliar no estado de São Paulo (17\%), ou ligeiramente superiores (9\%) às encontradas por Morellato et al. (2000) para a mata atlântica. As maiores proporções de espécies com dispersão anemocórica na encosta em regeneração podem estar relacionadas ao fato de se tratar de uma floresta jovem e ainda sofrer influência de ações antrópicas pretéritas, pois, de acordo com Van der Pijl (1982) e Howe e Smallwood (1982), a dispersão anemocórica ocorre, sobretudo, entre as espécies em estágio inicial de sucessão.

As três áreas de mata ciliar das cabeceiras do rio Grande apresentaram porte médio, com uma grande proporção de indivíduos (> 65\%) e espécies (> 66\%) com altura inferior a 17,5 m. Considerandose as espécies de pequeno e médio porte como características do estrato inferior (sub-bosque), e as espécies de grande porte, como representativas do estrato superior (dossel ou emergentes), verifica- 
se que, na mata ciliar de aluvião, aproximadamente $65 \%$ das espécies são típicas do estrato inferior, sendo que $52,66 \%$ dos indivíduos amostrados nessa área pertencem a essas espécies, enquanto $47,34 \%$ dos indivíduos e $34,57 \%$ das espécies pertencem ao estrato superior (Figura 4A e B), mesmo que haja uma proporção de imaturos no estrato inferior. Para a encosta em regeneração, aproximadamente $79,83 \%$ dos indivíduos e $87,99 \%$ das espécies pertencem ao estrato inferior e apenas $12 \%$ dos indivíduos e $20 \%$ das espécies pertencem ao estrato superior (Figura 4A e B). Já para a mata ciliar de encosta conservada, $54,75 \%$ dos indivíduos e $64 \%$ das espécies pertencem ao estrato inferior, enquanto $45 \%$ dos indivíduos e $36 \%$ das espécies pertencem ao estrato superior da floresta (Figura 4A e B).

A ocorrência elevada de arbustos e árvores de pequeno porte na área de encosta em regeneração sugere que a abundância de indivíduos nos diferentes estratos tenha sido influenciada pela diferença no estágio sucessional das áreas. Esse padrão foi também verificado por Tabarelli e Mantovani (1999) para florestas montanas em estádios iniciais de regeneração no estado de São Paulo.

As proporções de espécies nas guildas de dispersão variaram significativamente em relação às guildas de regeneração (Tabelas 2). O número de espécies pioneiras com dispersão primária abiótica (anemocórica e autocórica) foi maior do que o esperado para as três áreas estudadas. Verifica-se ainda que a anemocoria apresentou diferença significativa entre as guildas de regeneração. Nas duas áreas de encosta, verificou-se que houve diferenças significativas entre as guildas de regeneração e as guildas de dispersão. No entanto, na área de aluvião, constataram-se diferenças significativas apenas para as espécies pioneiras. Já para as espécies zoocóricas, houve uma predominância em todas as guildas de regeneração nas três áreas, apresentando valores para as espécies clímax tolerantes à sombras maiores do que os esperados somente para as duas áreas de encosta.

As proporções de espécies clímax tolerante à sombra variaram significativamente entre os estratos apenas nas duas áreas de encosta (Tabelas 2). Observa-se também que não houve diferença significativa em relação ao número de espécies anemocóricas entre os diferentes estratos. Mesmo assim, verifica-se uma tendência das espécies anemocóricas predominarem no estrato superior (dossel ou emergente), sendo maior do que o esperado nas três áreas. Já o número de espécies de pequeno e médio porte (estrato inferior ou sub-bosque), com síndrome de dispersão zoocórica, foi maior do que o esperado para as três áreas, porém, não diferiu estatisticamente das demais síndromes.

Comparando-se o número de espécies nas diferentes guildas de regeneração em relação aos estratos, percebe-se que houve diferença significativa nas duas áreas de encosta apenas para as espécies de pequeno e grande porte (Tabelas 2). No entanto, quando se compara o número de espécies registrado em cada guilda isoladamente, verifica-se que houve diferença significativa apenas para as espécies clímax exigente de luz na encosta em regeneração e clímax tolerante à sombra nas duas áreas (Tabelas 2).

A maioria das espécies anemocóricas registradas nas três áreas estudadas pertence ao estrato
A

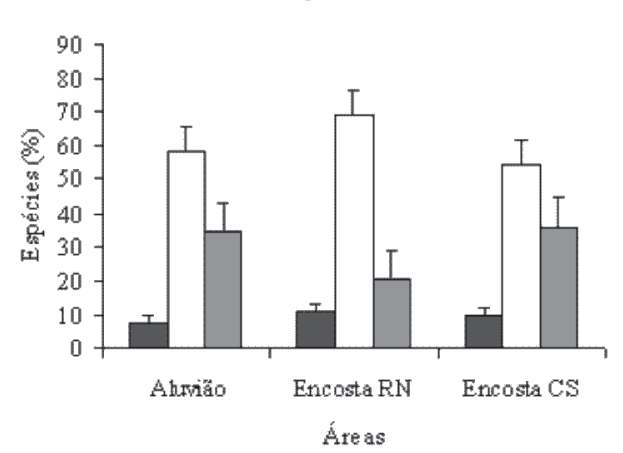

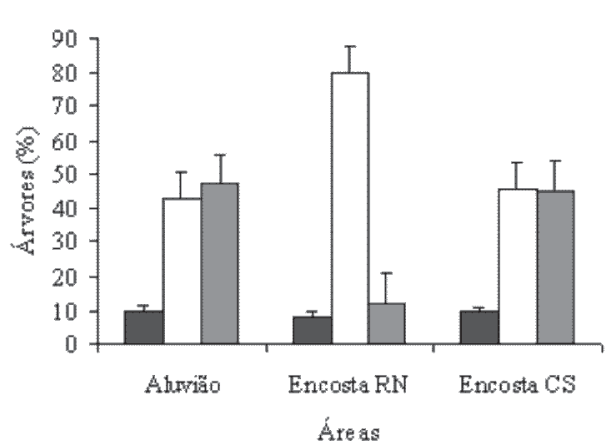

FIGURA 4: Distribuição das porcentagens de espécies (A) e árvores (B), por guilda de estratificação em três áreas de mata ciliar em Bocaina de Minas, MG. Em que: Peq = pequena; Med = média e Grd = grande.

FIGURE 4: Distribution of the percentage of species (A) and trees (B), for bedding guilds in three areas of ciliary forest in Bocaina of Minas, MG. In that: Peq: small, Med: average and Grd: big. 
Pereira, I. M. et al.

TABELA 2: Tabelas de contingência cruzando-se a distribuição das frequências observadas e esperadas (entre parênteses) das espécies arbóreas registradas em três áreas de mata ciliar nas cabeceiras do Rio Grande em Bocaina de Minas, MG, entre as guildas de dispersão, estratificação e regeneração. Os resultados dos testes de qui-quadrado são apresentados para cada tabela como um todo e, para suas linhas e colunas, em separado.

TABLE 2: Contingency tables crossing the distribution of the observed frequencies and expected (between parentheses) of the arboreal species registered in three areas of ciliary forest in the headboards of the Rio Grande in Bocaina of Minas, MG, among the dispersion guilds, bedding and regeneration. The results of the qui-square tests are presented for each table as a whole and separately for the lines and columns.

\begin{tabular}{|c|c|c|c|c|c|}
\hline \multirow{2}{*}{$\begin{array}{l}\text { Dispersão } \\
\text { Aluvião }\end{array}$} & \multicolumn{3}{|c|}{ Regeneração } & \multicolumn{2}{|c|}{ Qui-quadrado } \\
\hline & Pio & $\mathrm{CL}$ & $\mathrm{CS}$ & $\chi^{2}$ & $P$ \\
\hline Ane & $6(2,4)$ & $2(6,8)$ & $7(5,9)$ & 6,633 & $*$ \\
\hline Aut & $2(0,8)$ & $2(2,2)$ & $1(1,9)$ & 0,755 & ns \\
\hline Zoo & $5(9,7)$ & $32(27,11)$ & $24(24,1)$ & 2,597 & ns \\
\hline$\chi^{2}$ & 6,459 & 0,349 & 0,177 & 14,339 & \\
\hline$\stackrel{n}{P}$ & $* *$ & ns & ns & & $* *$ \\
\hline Encosta em Regeneração & Pio & $\mathrm{CL}$ & $\mathrm{CS}$ & $\chi^{2}$ & $P$ \\
\hline Ane & $11(4,5)$ & $13(11,2)$ & $0(8,3)$ & 15,637 & $* * *$ \\
\hline Aut & $3(1,3)$ & $2(3,3)$ & $2(2,4)$ & 1,274 & ns \\
\hline Zoo & $8(14,7)$ & $40(36,8)$ & $31(27,5)$ & 3,168 & ns \\
\hline Outras & $0(1,5)$ & $0(3,7)$ & $8(2,8)$ & 11,471 & $* * *$ \\
\hline$\chi^{2}$ & 43,364 & 445,043 & 341,074 & 24,640 & \\
\hline$\stackrel{M}{P}$ & $* * *$ & $* * *$ & $* * *$ & & $* * *$ \\
\hline Encosta Conservada & Pio & $\mathrm{CL}$ & CS & $\chi^{2}$ & $P$ \\
\hline Ane & $9(4,2)$ & $26(18,2)$ & $3(15,5)$ & 16,539 & $* * *$ \\
\hline Aut & $4(1,5)$ & $4(6,2)$ & $5(5,3)$ & 3,378 & $*$ \\
\hline Zoo & $11(17,4)$ & $73(74,4)$ & $72(63,9)$ & 2,945 & $\mathrm{~ns}$ \\
\hline Outras & $0(0,9)$ & $0(3,8)$ & $8(3,3)$ & 8,524 & $* *$ \\
\hline$\chi^{2}$ & 72,869 & 789,267 & 877,583 & 1,377 & \\
\hline$\stackrel{n}{P}$ & $* * *$ & $* * *$ & $* * *$ & & $* * *$ \\
\hline Dispersão & \multicolumn{3}{|c|}{ Estratificacão } & \multicolumn{2}{|c|}{ Qui-quadrado } \\
\hline Aluvião & Peq & Med & Grd & $\chi^{2}$ & $P$ \\
\hline Ane & $1(1,1)$ & $6(8,7)$ & $8(5,2)$ & 1,727 & $\mathrm{~ns}$ \\
\hline Aut & $1(0,4)$ & $2(2,9)$ & $2(1,7)$ & 0,131 & ns \\
\hline Zoo & $4(4,5)$ & $39(35,4)$ & $18(21,1)$ & 0,590 & ns \\
\hline$\chi^{2}$ & 0,182 & 0,886 & 1,381 & 4,650 & \\
\hline$\stackrel{n}{P}$ & $* * *$ & $* * *$ & $* * *$ & & ns \\
\hline Encosta em Regeneração & Peq & Med & Grd & $\chi^{2}$ & $P$ \\
\hline Ane & $1(2,4)$ & $14(13,4)$ & $9(3,3)$ & 8,819 & ns \\
\hline Aut & $1(0,7)$ & $6(3,9)$ & $0(0,9)$ & 0,917 & ns \\
\hline Zoo & $8(8,0)$ & $55(44,2)$ & $16(10,7)$ & 4,575 & $*$ \\
\hline Outras & $3(0,9)$ & $5(4,5)$ & $0(1,0)$ & 3,811 & $*$ \\
\hline$\chi^{2}$ & 67,651 & 690,993 & 235,136 & 32,826 & \\
\hline$\stackrel{R}{P}$ & $* * *$ & $* * *$ & $* * *$ & & $* * *$ \\
\hline Encosta Conservada & Peq & Med & Grd & $\chi^{2}$ & $P$ \\
\hline Ane & $4(3,9)$ & $16(20,7)$ & $18(13,4)$ & $2, \tilde{1} 15$ & ns \\
\hline Aut & $3(1,1)$ & $5(6)$ & $3(3,9)$ & 1,757 & ns \\
\hline Zoo & $12(16)$ & $89(84,9)$ & $55(55,1)$ & 0,907 & $\mathrm{~ns}$ \\
\hline Outras & $3(1,0)$ & $7(5,4)$ & $0(3,5)$ & 4,943 & $*$ \\
\hline$\chi^{2}$ & 105,389 & 1219,101 & 720,262 & 2,715 & \\
\hline$\stackrel{n}{P}$ & $* * *$ & $* * *$ & $* * *$ & & $* * *$ \\
\hline Dispersão & & egeneração & & $\mathrm{Qu}$ & \\
\hline Aluvião & Peq & Med & Grd & $\chi^{2}$ & $P$ \\
\hline $\mathrm{P}$ & $0(0,9)$ & $6(7,5)$ & $7(4,5)$ & 1,262 & ns \\
\hline $\mathrm{CL}$ & $4(3,0)$ & $23(23,8)$ & $14(14,2)$ & 0,082 & ns \\
\hline $\mathrm{CS}$ & $2(2,0)$ & $18(15,7)$ & $7(9,3)$ & 0,700 & ns \\
\hline$\chi^{2}$ & 0,418 & 0,362 & 1,263 & 3,941 & \\
\hline
\end{tabular}


TABELA 2: Continuação ...

TABLE 2: Continued ...

\begin{tabular}{lccccc}
\hline Dispersão & \multicolumn{3}{c}{ Regeneração } & \multicolumn{2}{c}{ Qui-quadrado } \\
\hline$P$ & $\mathrm{~ns}$ & $\mathrm{~ns}$ & $\mathrm{~ns}$ & & $\mathrm{~ns}$ \\
\hline Encosta em Regeneração & $\mathrm{Peq}$ & $\mathrm{Med}$ & $\mathrm{Grd}$ & $\chi^{2}$ & $\mathrm{~ns}$ \\
$\mathrm{P}$ & $1(1,6)$ & $16(12,8)$ & $5(7,6)$ & 1,179 & $\mathrm{~ns}$ \\
$\mathrm{CL}$ & $4(4,1)$ & $36(31,9)$ & $13(19,0)$ & 1,096 & $* * *$ \\
$\mathrm{CS}$ & $8(3,0)$ & $28(23,8)$ & $5(14,2)$ & 12,444 & \\
$\chi^{2}$ & 6,613 & 1,567 & 6,539 & 6,898 & $\mathrm{~ns}$ \\
$P$ & $\mathrm{~ns}$ & $\mathrm{~ns}$ & $\mathrm{~ns}$ & & $P$ \\
\hline Encosta Conservada & $\mathrm{Peq}$ & $\mathrm{Med}$ & $\mathrm{Grd}$ & $\chi^{2}$ & $\mathrm{~ns}$ \\
$\mathrm{P}$ & $2(1,8)$ & $13(13,9)$ & $9(8,3)$ & 0,061 & $*$ \\
$\mathrm{CL}$ & $6(7,6)$ & $48(59,8)$ & $49(35,6)$ & 6,961 & $* * *$ \\
$\mathrm{CS}$ & $14(6,5)$ & $56(51,1)$ & $18(30,4)$ & 12,534 & \\
$\chi^{2}$ & 7,688 & 2,522 & 9,346 & 17,250 & $* * *$ \\
$P$ & $*$ & $\mathrm{~ns}$ & $* *$ & & \\
\hline
\end{tabular}

Em que: Pio = pioneira, $\mathrm{CL}$ = clímax exigente de luz e CS = clímax tolerante à sombra; Ane: anecórica, Zoo: zoocórica, auto: autocórica e outras: outras formas de dispersão; Peq: pequena, Med: média e Grd: grande); $P=$ probabilidade; ns = não significativo; $*$ significativo a $10 \%$ de probabilidade, ${ }^{*}$ significativo a $5 \%$ de probabilidade $\mathrm{e}^{* * *}$ significativo a $1 \%$ de probabilidade.

superior (dossel e emergentes), enquanto a maioria das espécies zoocóricas pertence ao estrato inferior (sub-bosque). Esses resultados vão ao encontro do padrão já citado na literatura (MORELLATO e LEITÃO FILHO, 1992; CARMO e MORELLATO, 2001). De acordo com Howe \& Smallwood (1982) e Gentry (1983), a maior concentração de espécies anemocóricas nos estratos emergentes facilita a dispersão pelo vento. Já a concentração de espécies zoocóricas nos estratos inferiores da floresta parece estar relacionada à área de vida e atividades de animais dispersores (FOSTER, 1982).

De acordo com os resultados obtidos neste estudo para as três áreas, constatou-se que a flora arbustivo-arbórea das cabeceiras do rio Grande diferiu tanto em virtude do ambiente (aluvião e encosta) quanto do estádio de maturidade destas. Verificouse também que as espécies anemocóricas e autocóricas foram mais comuns no dossel das florestas, enquanto as zoocóricas foram mais comuns no sub-bosque.

Constatou-se, ainda, que as maiores proporções de espécies pioneiras, anemocóricas e de pequeno porte ocorreram em ordem decrescente para as áreas encosta em regeneração, aluvião e encosta conservada, evidenciando a existência de gradientes entre as áreas em relação ao estágio de sucessão ecológica destas.

\section{CONCLUSÕES}

De acordo com os resultados obtidos neste estudo para as três áreas de mata ciliar da região das cabeceiras do rio Grande em Bocaina de Minas, MG, pode-se concluir que: i) a flora arbustivo-arbórea das áreas diferiu tanto em virtude do ambiente (aluvião e encosta) quanto do estádio de maturidade das mesmas; ii) as espécies anemocóricas e autocóricas são mais comuns no dossel das florestas, enquanto as zoocóricas foram mais comuns no subbosque; iii) há uma elevada proporção de espécies e indivíduos zoocóricos, independentemente do ambiente e do estágio sucessional entre as florestas estudadas, em todas as circunstâncias; iv) as maiores proporções de espécies pioneiras, anemocóricas e de pequeno porte ocorreram em ordem decrescente para as áreas encosta em regeneração, aluvião e encosta conservada, evidenciando a existência de gradientes entre as áreas em relação ao estágio de sucessão ecológica destas; v) os modelos de implantação das matas ciliares das cabeceiras do rio Grande devem ser compostos por, no mínimo, $70 \%$ de espécies pioneiras e clímax exigente de luz, e, pelo menos, $60 \%$ destas devem ser zoocóricas.

\section{REFERÊNCIAS BIBLIOGRÁFICAS}

ANGEL-DE-OLIVEIRA, M. M. Aves que plantam: frugivoria e dispersão de sementes por aves. Boletim CEO, São Paulo, n. 13, p. 9-12, 1998.

BOtElho, S. A.; DAVIDE, A. C. Métodos 
silviculturais para recuperação de nascentes e recomposição de matas ciliares. In: SIMPÓSIO NACIONAL SOBRE RECUPERAÇÃO DE ÁREAS DEGRADADAS, 2002, Belo Horizonte. Anais... Belo Horizonte: UFLA/SOBRADE, 2002, p. 123-145.

CARMO, M. R. B.; MORELLATO, L. C. Fenologia de árvores e arbustos das matas ciliares da bacia do Rio Tibagi, estado do Paraná, Brasil. In: RODRIGUES, R. R.; LEITÃO FILHO, H F. (Eds.) Matas ciliares: Conservação e Recuperação. 2. ed. São Paulo: Ed. da Universidade de São Paulo/Fapesp, 2001, p.125-141.

DENSLOW, J. S. Gap partioning among tropical rain forest trees. Biotropica, Lawrence, v. 12, n. 2, p. 47-55, 1980.

DENSLOW, J. S. Patterns of plant diversity during succession under different disturbances regimes. Oecologia, Wisconsin, v. 46, n. 1, p. 18-21, 1980. DURIGAN, G.; SILVEIRA, E. R. Recomposição da nata ciliar em domínio de cerrado, Assis, SP. Scientia Forestalis, Piracicaba, v. 1, n. 56, p. 135-144, 1999. FOSTER, R. B. The seasonal rhythm of fruitfall on Barro Colorado Island. In: E.G. Leigh-Junior; A.S. Rand; D. M. Windsor. The ecology of a tropical forest. Washington: Smithsonian Institution Press, 1982.

GARCIA, R. J. F.; PIRANI, J. R. Estudo florístico dos componentes arbóreo e arbustivo da mata do Parque Santo Dias, São Paulo, SP, Brasil. Boletim de Botânica da Universidade de São Paulo, São Paulo, v. 19, p. 15-42, 2001.

GENTRY, A. H. Patterns of neotropical plant species diversity. Evolution Biology, v. 15, p. 1-84, 1982.

GENTRY, A. H. Dispersal ecology and diversity in neotropical forest communities. Sonderbände Naturwissenschaftlichen Vereins im Hamburg, v. 7, p.303-314, 1983.

GÓMEZ-POMPA, A. A. Posible papel de la vegetación secundaria en la evolución de la flora tropical. Biotropica, Lawrence, v. 3, n. 2, p. 125 135. 1971

GUEDES, M. C. et al. Seleção de espécies para recuperação de áreas degradadas por meio de ilhas de vegetação. In: SIMPOSIO NACIONAL DE RECUPERAÇÃO DE ÁREAS DEGRADADAS, 1997, Viçosa. Anais... Viçosa: SOBRADE/UFV. 1997, p. 279-282.

HOWE, H. F.; SMALLWOOD, J. Ecology of seed dispersal. Annual Review of Ecology and Systematic, v. 13, p. 201-228, 1982.
KLEIN, R. M. Ecologia da flora e vegetação do vale do Itajaí. Sellowia, Itajaí, v. 32, p. 165-389, 1980. LOISELLE, B. A. et al. Spatial and temporal variation of seed rain in a tropical lowland wet forest. Biotropica, Lawrence, v. 28, n. 1, p. 82-95, 1996. LORENZI, H. Árvores brasileiras: Manual de identificação e cultivo de plantas arbóreas nativas do Brasil. Nova Odessa: Plantarum, 2002a. v. 1.

LORENZI, H. Árvores brasileiras: Manual de identificação e cultivo de plantas arbóreas nativas do Brasil. Nova Odessa: Plantarum, 2002b. v. 2. MARQUES, J. Q. A. et al. As perdas por erosão do Estado de São Paulo. Bragantia, Campinas, v. 20, p. 1143-1181, 1961.

MARTINEZ RAMOS, M.; SOTO CASTRO, A. Seed rain and advanced regeneration in a tropical rain forest.Vegetatio, v. 108, n. 1, p. 299-318, 1993. MARTINS, S. E. et al. Síndromes de dispersão de espécies arbóreas da Mata Atlântica de encosta no estado de São Paulo. In: CONGRESSO NACIONAL DE BOTÂNICA, 46., 1995, Ribeirão Preto. Anais... Ribeirão Preto, 1995. p. 167.

MORELLATO, L. P. C.; LEITÃO FILHO, H. L. F. Padrões de frutificação e dispersão na Serra do Japi. In: MORELLATO, L. P. C. História natural da Serra do Japi: ecologia e preservação de uma área florestal no Sudeste do Brasil. Campinas:UNICAMP/ FAPESP, 1992

MORELLATO, L. P. C. et al. Estudo comparativo da fenologia de espécies arbóreas de floresta de altitude e floresta mesófila semidecídua na Serra do Japí, Jundiaí, São Paulo. Revista Brasileira de Botânica, São Paulo, v.12, n.1-2, p.85-98, 1989. MORELLATO, L. P. C. et al. Phenology of Atlantic rain forest trees: a comparative study. Biotropica, Lawrence, v.32, n.4b, p.811-823, 2000.

NUNES, Y. R. F. et al. Variações da fisionomia, diversidade e composição de guildas da comunidade arbórea em um fragmento de floresta semidecidual em Lavras, MG. Acta Botânica Brasílica, São Carlos, v.7, n.2, p.213-229, 2003.

OLIVEIRA-FILHO, A. T. Estudos ecológicos da vegetação como subsídios para programas de revegetação com espécies nativas: uma proposta metodológica. Cerne, Lavras, v. 1, n. 1, p. 64-72, 1994.

OLIVEIRA-FILHO, A. T. et al. Effects of past disturbance and edges on tree community struture and dynamics within a fragment of tropical semidecidous Forest in South-eastern Brazil over a five-year period (1987-1992). Plant Ecology,

Ci. Fl., v. 20, n. 2, abr.-jun., 2010 
Dordrecht, v. 131, n. 1, p. 45-66, 1997.

PENHALBER, E. de. F.; MANTOVANI, W. Floração e chuva de sementes em mata secundária em São Paulo, SP. Revista Brasileira de Botânica, São Paulo, v. 20, n. 2, p. 205-220, 1997.

PESSOA, S. V. A. et al. Composição florística e estrutura do componente arbustivo-arbóreo de um trecho secundário de floresta montana na Reserva Ecológica Macaé de Cima. In: LIMA, H. C.; GUEDES-BRUNI, R. R. (Eds.). Serra de Macaé de Cima: diversidade florística e conservação em Mata Atlântica, Jardim Botânico do Rio de Janeiro, Rio de Janeiro, 1997.

REIS, M. S. Dispersão de sementes Euterpe edulis (Palmae) em uma Floresta Ombrófila Densa Montana da Encosta Atlântica em Blumenau, SC. 1995. 154 f. Tese (Doutorado em Biologia Vegetal), Universidade de Campinas, Campinas, 1995.

RODRIGUES, R. R.; GANDOLFI, S. Recomposição de florestas nativas: princípios gerais e subsídios para uma definição metodológica. Revista Brasileira de Horticultura Ornamental, Campinas, v. 2, n. 1, p. 4-15,1996.
SWAINE, M. D.;WHITMORE, T. C. On the definition of ecological species groups in tropical rain forest. Vegetatio, v. 75, n. 1-2, p. 81-86, 1988. TABARELLI, M.; MANTOVANI, W. A. Regeneração de uma floresta tropical Montana após corte e queima (São Paulo-Brasil). Revista Brasileira de Biologia, São Carlos, v. 59, n. 2, p. 239-250, 1999.

VAN DER PIJL, L. Principles of dispersal in higher plants. Berlin, Springer-Verlarg, 1982.

ZAR, J. H. Biostatistical analysis, New Jersey: Prentice Hall, 1996

WHEELWRIGTH, N. T. Competition for disperses, and th timing of floweing and fruiting in a guild of tropical trees. Oikos, v. 44, p. 465-477, 1985.

WHITMORE, T. C. On pattern and process in forest. Pp.45-59. In:, E.T. Newman (ed.). The plant community as a working mechanism . Oxford: Blackwell, 1982.

WILSON, J. B. A null model of guild proportionality, applied to stratification of new Zealand temperate rain forest. Oecologia, Wisconsin, v. 80, n. 2, p. 263267, 1989. 